"This is a pre-print of an article published in Current Medicinal Chemistry. The final authenticated version is available online at: https://doi.org/10.2174/0929867325666180508094637 ”.

"Esta es una preimpresión de un artículo publicado en Current Medicinal Chemistry. La versión final autenticada está disponible en línea en: https://doi.org/10.2174/0929867325666180508094637 ".

\title{
Genetically Engineered Elastin-based Biomaterials for Biomedical Applications
}

\author{
Mercedes Santos, Sofía Serrano-Dúcar, Juan González-Valdivieso, Reinaldo Vallejo, Alessandra Girotti, \\ Purificación Cuadrado and Francisco Javier Arias*
}

BIOFORGE Research Group, CIBER-BBN, University of Valladolid, 47011 Valladolid, Spain

\begin{abstract}
Protein-based polymers are some of the most promising candidates for a new generation of innovative biomaterials as recent advances in genetic-engineering and biotechnological techniques mean that protein-based biomaterials can be designed and constructed with a high degree of complexity and accuracy. Moreover, their sequences, which are derived from structural protein-based modules, can easily be modified to include bioactive motifs that improve their functions and material-host interactions, thereby satisfying fundamental biological requirements.

The accuracy with which these advanced polypeptides can be produced, and their versatility, self-assembly behavior, stimuli-responsiveness and biocompatibility, means that they have attracted increasing attention for use in biomedical applications such as cell culture, tissue engineering, protein purification, surface engineering and controlled drug delivery.

The biopolymers discussed in this review are elastin-derived protein-based polymers which are biologically inspired and biomimetic materials. This review will also focus on the design, synthesis and characterization of these genetically encoded polymers and their potential utility for controlled drug and gene delivery, as well as in tissue engineering and regenerative medicine.
\end{abstract}

Keywords: drug delivery, tissue engineering, Elastin-like Recombinamers, nanocarriers, hydrogels, stimuli-responsive, monomers, self-assembly

\section{INTRODUCTION}

Novel biomaterials with innovative and advanced properties [1] have found specific applications in the field of biomedicine and can play a particularly important role in areas such as controlled drug delivery or tissue engineering, as well as regenerative medicine in general [2-3]

New biomaterials are needed for specific use as vehicles for the release of therapeutic molecules in controlled drug delivery. Indeed, as only a small fraction of drugs administered reach their target tissues, improving the efficacy of therapeutic agents has become an important objective for researchers. Moreover, the efficacy of traditional chemotherapeutic drugs is limited by their adverse effects on non-target cells. Thus, these new kinds of materials can provide significant advantages as they are designed to lower the toxicity and improve the solubility, stability and pharmacokinetics of conventional drugs [4] with respect to the toxic effects and tissue damage provoked by administration of the free drug [5]. Advanced drug- delivery systems (DDSs) are designed to control the release of drugs into target tissues by responding to certain stimuli, thus reducing the dose of the drugs and their related side effects [6]. As such, advanced biomaterials capable of undergoing bioresponsive self-assembly and higher-order supramolecular organization will be required.

The field of biomedicine is also trying to develop novel biomedical devices in order to improve the quality of life of patients who have suffered specific tissue or organ damage. Such devices should act as physical supports for the healing process and should also be able to interact actively with biological mechanisms and preserve or enhance tissue functions [7-8]. The development of these systems tends to occur synergistically with the acquisition of a better understanding of the mechanisms that govern the function of body compartments and of human genetics, thus allowing the use of advanced approaches that mimic naturally occurring constructs in an attempt to emulate the biological and mechanical properties of the target tissue while ensuring 
adequate biocompatibility and biodegradability of the scaffold. Advanced approaches try to emulate natural tissues by considering them to comprise a compendium of porous 3D networks made up of biomaterials, cells and bioactive molecules, such as growth factors, which, together, promote the regeneration of damaged tissues or organs. In this regard, the physical building blocks utilized in tissue engineering must be as inherently safe and as similar to nature as possible [9]. Given their porous 3D structures and their ability to encapsulate cells and biomolecules and to control their release, hydrogels are the most widely used scaffolds for the regeneration and replacement of natural soft tissues [10].

Biomedical devices for drug delivery and tissue repair can be synthetic or natural and, depending on the application, degradable or not. Protein-based biopolymers inspired by naturally occurring proteins in the extracellular matrix (ECM) are excellent candidates as key components of advanced drug-delivery devices and enhanced substitutes in regenerative medicine as their properties are determined by the physicochemical features and sequence of their constituent monomers. Despite the significant progress made in the field of synthetic polymers as regards increases in the efficiency of polymerization techniques and lower polydispersities, genetic engineering still provides unparalleled control over the component macromolecules used to build delivery carriers and tissue substitutes. From the perspective of sequence-structure relationships, genetically engineered drug carriers present different macro-, micro-, or nanoscale properties with differences in the length and composition of the amino acid sequence. Examples of well-developed genetically engineered drug carriers and customized supports related to tissue repair include protein polymers comprising from elastin-like polypeptides [11].

Elastin-like polypeptides (ELPs) are bioinspired polymers comprising short pentapeptide repeats found in natural elastin, with one of the most common being the pentapeptide sequence VPGXG, where X can be any amino acid except L-proline. A new term, namely elastin like recombinamers (ELRs), has been created to refer to those ELPs bioproduced using genetic-engineering techniques. DNA technology allows ELRs to be produced with complete control over the amino acid sequence, and with the possibility of including numerous different functionalities and bioactive sequences [12]. As they are biologically inspired biomaterials, ELRs present characteristic features that make them an interesting alternative for the development of novel biomedical devices because of their biological and mechanical properties, such as biocompatibility, biodegradability, and thermally and environmentally responsive behavior. Moreover, ELRs exhibit an inverse temperature transition (ITT) such that, below a characteristic temperature, known as the transition temperature $(\mathrm{Tt})$, they remain soluble in a random coil conformation, self-assembling hydrophobically above this $\mathrm{Tt}$, thus resulting in the reversible formation of coacervates as a result of a conformational reorganization at the molecular level. Coacervation of the polymer backbone can be triggered by multiple factors, including variations in temperature, $\mathrm{pH}$, light, concentration of ionic species, etc. In addition, their stimulus-responsive behavior can be tuned as $\mathrm{Tt}$ is controlled by the composition and molecular weight of the recombinamer [13]. Given their cell-friendly behavior, tunable mechanical properties, thermal sensitivity, and ability to self-assemble, they are useful materials for the most cutting-edge applications in the fields of nanotechnology and biomedicine [14-15] and, specifically, for controlled drug delivery [16] and tissue engineering.

In this paper we aim to review applications of ELRs as advanced drug-delivery systems that can safely carry therapeutic agents to specific sites. The variable sequences, architectures and assemblies of ELRs allow different drugrelease devices, ranging from monomeric units to nanoparticles and 3D assemblies, to be designed. The use of monomeric ELRs as soluble delivery systems, either fused or conjugated to chemicals or proteins, including targeting peptides as receptor ligands or cell-penetrating domains, will also be reviewed. Such systems are designed to selfassemble in response to external stimuli, thus making them suitable for systemic delivery and especially useful in tumor tissue treatments thanks to their thermoresponsive behavior. Similarly, the ability of ELRs to self-assemble means that they can form nanoparticles (NPs). As such, the applications of NPs as drug nanocarriers when presenting growth factors, proteins, or therapeutic agents on their surfaces, or those designs that are able to internalize the drug, therapeutic agent, or even DNA such that permeability and retention effects can be enhanced, will be reviewed, along with the use of hydrogels and depots as 3D platforms for drug-delivery systems. Both physical and chemical ELR chain crosslinking allow the formation of macroscopic scaffolds that are able to overcome issues related to inappropriate drug concentration loading, thereby allowing controlled localization of the bioactive agent release site and rate. The last section is devoted to different approaches to the manufacture of ELRbased customised tissue engineered supports for the regeneration of tissues, with a particular focus on skeletal and vascular systems..

\section{ADVANCED DRUG DELIVERY DEVICES FROM ELRs}

In this chapter, the different structures of ELRs are studied as the fundamentals on the construction of diverse drug delivery devices. In the first and second subchapter, devices from monomeric ELRs to amphiphilic ELRs, able to form nanoparticles, has been covered. The chapter finishes with macroscopic ELRs for drug delivery in form of depots or as hydrogels.

\subsection{Monomeric ELRs as drug nanocarriers}

As many pharmaceutical molecules exhibit short halflives due to the fact that their small size results in fast glomerular filtration, ELRs have been proposed as possible drug carriers because they increase the size and stability of 
their load. Monomeric elastin-like recombinamers are simple elastin-based polypeptides that can be adapted for use as therapeutic agents. Thus, they can be used as soluble delivery systems when fused to therapeutic proteins in a recombinant manner, or as pharmacokinetic enhancers, either by chemical conjugation or by fusion protein expression of the drug [17]. A monomeric ELR carrier allows the pharmacokinetics and biodistribution of its drug load to be improved when compared to the free drug, thus resulting in specific delivery of the therapeutic agent [18]. Moreover, the genetic engineering of elastin-like recombinamers allows targeting peptides, such as receptor ligands or cell-penetrating domains, to be incorporated and reactive sites useful for covalent chemical conjugation of drugs to be included into the ELR sequence [19]. Similarly, ELRs can be designed to self-assemble in response to an external stimulus, thus making them suitable for systemic delivery of a therapeutic drug and local delivery of the therapeutic agent into the damaged tissue [20]. Useful therapeutic drug-delivery systems comprise a monomeric ELR conjugated to a molecule with known therapeutic activity. Polymer drug conjugation involves attaching a selected drug to a macromolecule, with synthetic chemicals and proteins being the most popular candidates. The recombinant origin of ELRs allows the incorporation of reactive residues, such as the thiol group in cysteines or amine group in lysines, which can be used for further bioconjugation of different therapeutic drugs. If the load is a protein, the ELR-load construct can be prepared by genetic engineering.

Although cancer is the most common disease in the world, many therapeutic agents used against cancer are hydrophobic compounds with a molecular weight of less than $500 \mathrm{Da}$ that have significant systemic toxicity, suboptimal pharmacokinetics, and a low therapeutic index, defined as the ratio between the dose needed to cause death in $50 \%$ of a population (lethal dose) and the smallest dose able to induce the desired effect in 50\% of a population (effective dose). Moreover, several chemotherapeutic drugs, such as Paclitaxel, Doxorubicin, Camptothecin or Melphalan, with anti-cancer properties exhibit an important disadvantage, namely toxicity in normal healthy cells. These properties are an important motivation for investigating the potential use of elastin-like recombinamer-based systems as soluble macromolecular carriers for conjugated anticancer drug delivery to take advantage of their favorable pharmacokinetic profile [21].

ELRs are anti-cancer drug-delivery systems with a good perspective for future applications that take advantage of their thermal properties for therapeutic hyperthermia. Hyperthermia consists of raising the local, regional, or whole body temperature from physiological $\left(37^{\circ} \mathrm{C}\right)$ to approximately $42^{\circ} \mathrm{C}$. Elastin-like recombinamers are attractive as carriers for drug delivery because they are disordered and water-soluble below a characteristic transition temperature ( $\mathrm{Tt}$ ) but, when the temperature increases above this Tt, ELRs aggregate and accumulate. The phase transition of these polypeptides could be exploited for use in localized drug delivery to tissues because ELRs are soluble at a body temperature of $37^{\circ} \mathrm{C}$, thus meaning that they can freely circulate throughout the body, but become insoluble when circulating in overheated tumor tissue, thus accumulating in the tumor, where hyperthermia means that the tissue is warmer than the transition temperature (Tt). When therapeutic hyperthermia is used, higher concentrations of ELR-drug conjugates should be observed at the heated sites. As such, hyperthermia could increase the efficient delivery of ELR conjugates to tumor tissue as a result of tumor vasculature permeability and perfusion.

The plasma membrane is one of the most difficult problems that a drug delivery system has to face. The plasma membrane is a permeable barrier present in living cells that is essential for cell survival and function. Thus, the passage of a bioactive molecule through the plasma membrane is the main difficulty that must be overcome to ensure intracellular delivery of a drug. Small molecules can cross this membrane by direct diffusion through the lipid bilayer, whereas proteinbased drugs enter cells by membrane mobile transport. However, the efficient entry of a therapeutic agent could be enhanced by a variety of vectors, with cell-penetrating peptides (CPPs) being one of the most popular and efficient for achieving cellular uptake [22]. The cellular uptake of ELRs can be simplified by functionalizing the ELR with a $\mathrm{CPP}$, thereby promoting non-specific cellular uptake of the load via endocytic pathways. CPPs are peptides comprising 5-30 amino acids that can cross the cellular membrane. As such, different CPPs have been incorporated into ELR-drug bioconjugates to enhance the specific release of anticancer drugs in the tumor. Moreover, the use of CPPs allows the rapid degradation of these drugs and their poor tumor cell penetration to be overcome. The first CPP was reported in 1988 by Frankel and Green, who found that the HIV transactivator of transcription (TAT) protein could cross cell membranes and was efficiently internalized in vitro [23-24].

In this section it has been described the drug delivery behavior of monomeric ELRs depending on the molecule attached, either bioconjugated drugs or bioactive peptides forming fusion proteins.

\subsubsection{Drugs bioconjugated to monomeric ELRs:}

The chemotherapeutic agent Doxorubicin is one of the most widely studied molecules in bioconjugation to ELRs. As one of the most interesting features of ELRs is their responsiveness to thermal conditions, Doxorubicin has been chemically bioconjugated to different ELRs to ensure its accumulation in the tumor. Thus, in vitro studies of the cytotoxicity and localization of the drug in squamous cell carcinoma compared the free and conjugated drug. Although the cellular death level was not statistically significantly different when comparing the free drug and the conjugated drug, the different location of the drug in the cell was demonstrated. Thus, the ELR-Doxorubicin conjugate was shown to be dispersed in the cytoplasm, exhibiting a different cell death pathway than the free drug, which is internalized into the cell nucleus [25]. Similarly, Saxena et al. also proved that Doxorubicin and an ELR-Doxorubicin conjugate exhibit a similar cell killing ability even though 
the free drug and the conjugate show different intracellular distribution patterns [26].

In this line, and exploiting CPP strategy, Walker et al. conjugated Doxorubicin to the free cysteine of an ELR by way of a denaturing process comprising the addition of tris(2-carboxyethyl)phosphine (TCEP) to the polypeptide and subsequent addition of Doxorubicin. The authors used different CPP sequences in order to inhibit tumor growth in mice and demonstrated delivery of the CPP-ELRDoxorubicin construct into the cell nucleus in MCF-7 breast carcinoma cells using fluorescence microscopy experiments. When hyperthermia was applied, this construct was found to exhibit tumor inhibition in a 2:1 ratio when compared with free Doxorubicin, thus showing that an ELR is able to aggregate and accumulate in solid tumors in response to hyperthermia while remaining soluble under non-pathogenic physiological conditions [27].

Similar studies have used Doxorubicin conjugated to a cysteine residue of a SynB1-ELR. The cell-penetrating peptide SynB1 is derived from protegrins from porcine leukocytes and is known to be able to cross the cell membrane by endocytosis. Consequently, the SynB1 cellpenetrating peptide was included in the ELR to enhance cellular entry of the construct and allowed release of the drug from the conjugate, showing an intranuclear distribution of Doxorubicin compared with nonconjugated labeled Doxorubicin. It is noticeable that when hyperthermia was applied, the SynB1-ELR-Doxtreated group showed a twofold increase in the tumor accumulation of Doxorubicin compared with Syn-B1ELR-Dox without hyperthermia. Moreover, a similar increase was seen with respect to free Doxorubicin for the hyperthermia group. The authors tested this construct in an E0771 syngeneic mouse breast cancer model, where the construct based on SynB1-ELRDoxorubicin was used in combination with a hyperthermia treatment that involved heating the tumor with a laser emitting diode device. The results showed that mice treated with SynB1-ELR-Dox combined with hyperthermic treatment had a 2.5-fold smaller tumor volume than the group of SynB1-ELP-Dox treatment with no thermal inducement at 14 days post-treatment [28]. In the same way, Moktan et al. coupled paclitaxel, an apoptosis-inducing drug, to a SynB1-ELR construct via the reactive thiol group on a cysteine residue. They then tested the construct in vitro in MCF-7 breast cancer cell culture and the results showed that treatment under hyperthermia conditions $\left(42^{\circ} \mathrm{C}\right)$ was able to kill $50 \%$ of the MCF-7 cells [29].

\subsubsection{Fused Recombinamers as bioactive peptides carriers:}

A second approach for obtaining therapeutic monomeric ELRs involves the construction of fusion recombinamers, in which a protein is attached to an elastin-like recombinamer to improve its expression and delivery. The main problem as regards the efficiency of drug-delivery systems is drug resistance. As such, the incorporation of mechanisms to facilitate release of the drug in the cytoplasm could be the best strategy for overcoming this drawback. The use of genetic engineering allows the addition of different targeting peptides, such as cell-penetrating domains or receptor ligands, thus providing ELRs with one or more copies of a targeting molecule and improving the cellular uptake of constructs with a therapeutic function in damaged tissues.

An AP1-based recombinamer has been developed by Sarangthem et al. by fusing six units of AP1, a ligand for the IL-4 receptor overexpressed in cancerous cells that enhances the expression of some anti-apoptotic proteins, to an ELR. The resulting construct was shown to bind to the IL-4 receptor in vitro and in vivo and to accumulate in the tumor [30].

One disadvantage of therapeutic proteins is their short circulating half-lives, thus meaning that the frequent administration of high concentrations is required to achieve therapeutic levels. As an example, recombinant human interferon alpha (IFN- $\alpha$ ) has been used to treat viral diseases or several types of cancer. Hu et al. have developed a new protein-ELR fusion delivery platform to extend the circulating half-life of IFN- $\alpha$. Thus, the addition of IFN- $\alpha$ at the C-terminus reduced the bioactivity of IFN- $\alpha$ and also improved its pharmacokinetics. In vivo experiments showed higher levels of IFN- $\alpha$-ELP in tumor, heart, kidney, liver, spleen, lung and pancreas than of IFN- $\alpha$ alone. The administration of IFN- $\alpha$-ELR to mice with 30-day-old ovarian tumors almost completely inhibited tumor growth, while treatment with IFN- $\alpha$ inhibited tumor growth only slightly. As a result, animal survival was increased from 42 days for mice receiving IFN- $\alpha$ treatment to 87 days when the IFN- $\alpha$-ELR treatment was applied. Moreover, blood biochemistry and hematological studies showed no significant toxicity for either treatment [31].

In other cases, cell-penetrating peptides have been fused to fusion proteins, such as the SynB1-ELR1-KLAK fusion polypeptide developed by Moktan et al., which contains the SynB1 cell-penetrating peptide and the KLAK peptide (known to induce apoptosis by disrupting the mitochondria). These authors demonstrated that hyperthermia enhanced the cytotoxic effect and cellular uptake of the SynB1-ELR1KLAK construct. Moreover, confocal microscopy experiments showed that the fusion polypeptide was localized in the cytoplasm [32].

Several groups have developed different ELR fusion constructs containing the cell-penetrating peptide Tat (derived from the trans-activating protein found in HIV-1). The resulting Tat-ELR was able to inhibit adhesion, invasion and migration of ovarian cancer cells [33]. Moreover, this ELR fusion seems to have anti-metastatic potential in vivo in an ovarian cancer metastasis model.

A further example is the construct p21-ELR-Bac, which presents the cell-penetrating peptide $\mathrm{Bac}$ at the $\mathrm{C}$-terminus and the cell cycle inhibitory peptide p21 at the $\mathrm{N}$-terminus and was developed from an earlier construct (with the Bac cell-penetrating peptide at the $\mathrm{N}$-terminus) reported in a previous study [34]. This new construct was used against pancreatic cancer by applying hyperthermia in vitro (cell culture at $42{ }^{\circ} \mathrm{C}$ ) and in vivo. The aim of this study was to 
lower the dose of the drug while enhancing the anti-tumor effect. The new configuration killed tumor pancreatic cancer cells (S2013, Mia PaCa-2 and Panc-1) in a dose-dependent manner, and hyperthermia enhanced the cytotoxicity of the ELR-conjugate. The three different cell lines tested (S2013, Mia PaCa-2 and Panc-1) showed significant increases in cell cycle S-phase arrest population and decreased active Sphase. Thus, this construct can reach the cellular nucleus and arrest the cell cycle. In vivo studies demonstrated that twice as much of the construct accumulated when combined with therapeutic hyperthermia when compared with the absence of hyperthermia. The results showed hyperthermia-induced aggregation of the conjugates $\left(42{ }^{\circ} \mathrm{C}\right)$ and a higher cellular uptake rate in comparison with physiological body temperature. Furthermore, addition of the cell-penetrating peptide Bac reduced the plasma half-life of p21-ELP-Bac in heated tumors to 3.25 hours from 8.7 hours in the case of unmodified ELR. Once the mechanism of action of the p-21ELR-Bac construct had been determined, gemcitabine pretreatment was added to study its effect on cell proliferation. To that end, cells were treated with the nucleoside analog gemcitabine for 24 hours, then the medium was removed and the cells incubated with p-21ELR-Bac. The combination of gemcitabine treatment with p21-ELR-Bac resulted in a synergistic inhibitory effect on cell proliferation in in vitro studies. In vivo studies showed that combination of the construct with gemcitabine resulted in greater tumor inhibition (81\%) than p-21-ELR-Bac (34\%) or gemcitabine (58\%) alone. In conclusion, the combinatory effect of gemcitabine with an elastin-like polypeptide may offer a promising strategy for reducing drug dose and toxicity [35].

Recently, Mikecin et al. tested the combination of Bortezomib, a proteasome inhibitor, with p21-ELR-Bac in the prostate cancer cell lines DU-145 and PC-3 and showed that the combined treatment increased cell-cycle arrest and apoptosis with respect to single treatments [36]. Similarly, Bidwell et al. studied the thermal properties of the construct Bac-ELR1-H1, which contains the c-Myc inhibitory peptide H1 based on the sequence of helix 1 from the helix-loophelix domain from the oncogenic protein c-Мус, against breast cancer. Progressive accumulation of the construct in the tumor vasculature and tissue was observed after applying four cycles of heating and cooling. This hyperthermia treatment resulted in an efficient inhibition of tumor growth (70\%) in comparison with Bac-ELR1-H1 treatment without hyperthermia or the thermally non-responsive Bac-ELR2-H1 construct. ELR2 has a similar composition and molecular weight to ELR1 but does not aggregate under the mild hyperthermia temperatures used by Bidwell et al. [37].

\begin{tabular}{|c|c|c|c|}
\hline \multirow{2}{*}{ Disease } & $\begin{array}{c}\text { Molecule } \\
\text { attached }\end{array}$ & $\begin{array}{c}\text { Mechanism of } \\
\text { action }\end{array}$ & Reference \\
\hline \multirow{4}{*}{ Breast cancer } & AP1 & $\begin{array}{c}\text { IL4-receptor } \\
\text { ligand }\end{array}$ & Sarangthem et al. [30] \\
\cline { 2 - 5 } & Doxorubicin & Intercalating drug & Walker et al. [27] \\
\cline { 2 - 4 } & Doxorubicin & Intercalating drug & Moktan et al. [28] \\
\cline { 2 - 4 } & H1 & c-Myc inhibitor & Bidwell et al. [37] \\
\cline { 2 - 4 } & KLAK & $\begin{array}{c}\text { Mitochondria } \\
\text { disrupter }\end{array}$ & Moktan et al. [32] \\
\hline
\end{tabular}

\begin{tabular}{|c|c|c|c|}
\hline & Paclitaxel & $\begin{array}{l}\text { Apoptotic-inducer } \\
\text { drug }\end{array}$ & Moktan et al. [29] \\
\hline \multirow[b]{2}{*}{ Ovarian cancer } & IFN- $\alpha$ & $\begin{array}{c}\text { Th1-response } \\
\text { enhancer }\end{array}$ & Hu et al. [31] \\
\hline & Tat & $\begin{array}{l}\text { Cell penetrating } \\
\text { peptide }\end{array}$ & Raucher et al. [33] \\
\hline \multirow{2}{*}{$\begin{array}{l}\text { Pancreatic } \\
\text { cancer }\end{array}$} & p21 & $\begin{array}{l}\text { Cell cycle } \\
\text { inhibitor }\end{array}$ & Ryu et al. [34] \\
\hline & p21 & $\begin{array}{l}\text { Cell cycle } \\
\text { inhibitor }\end{array}$ & Walker et al. [35] \\
\hline Prostatic cancer & $\mathrm{p} 21$ & $\begin{array}{l}\text { Cell cycle } \\
\text { inhibitor }\end{array}$ & Mikecin et al. [36] \\
\hline $\begin{array}{l}\text { Renovascular } \\
\text { disease }\end{array}$ & VEGF & Growth factor & Chade et al. [38] \\
\hline $\begin{array}{l}\text { Squamous } \\
\text { carcinoma }\end{array}$ & Doxorubicin & Intercalating drug & Saxena et al. [26] \\
\hline
\end{tabular}

Table 1. Molecules attached to monomeric elastin-like recombinamers and their mechanism of action.

Although many ELR-based studies have focused on cancer, biopolymers can be used in other disease treatments. As an example, Chade et al. developed an ELR-VEGF fusion protein against renovascular disease (RVD). Progressive damage of the stenotic kidney is mediated by decreased expression of renal vascular endothelial growth factor (VEGF), thus restricting renal angiogenesis, with VEGF playing a pivotal role. The in vitro and in vivo results showed that the construct was able to stimulate proliferation, migration, and tube formation in cells. The ELR-VEGF accumulated in the kidney, where it was able to reduce renal injury and improve renal function in the stenotic kidney by promoting the generation of new cortical and medullary microvessels. When compared with unconjugated VEGF therapy, the ELR-VEGF fusion did not alter the potency of VEGF, but showed a prolonged half-life and was more effective at recovering renal function. Thus, the ELR-VEGF strategy could be applied in other models of acute and chronic renal disease [38].

\subsubsection{Monomeric ELRs as Nanoparticle Coatings}

Although the main use of monomeric ELR constructs is drug delivery itself, another interesting approach for systems based on these monomers is their use to coat therapeutic nanoparticles to evade the immune system.

Elastin-like recombinamers are known to be nonimmunogenic and biodegradable, thus meaning that they can potentially be used to assemble nanoparticles coated with short recombinamers. Thus, given the minimal immune response triggered by their amino acid sequence, the lack of immunogenicity makes these polymers very good candidates as carrier systems. Recent reports have demonstrated that repeated injections of PEG-coated nanoparticles in mice and rats can provoke the formation of anti-PEG antibodies and their aggregation and opsonization by antigen-presenting cells, such as macrophages or dendritic cells, thus enhancing the immune response against PEGylated nanoparticles [39]. ELRs are characterized by being biocompatible and invisible to the immune system. Taking advantage of one of the main features of short recombinamers, Noury et al. have recently 
developed an ELR-based system that can be used to hide DNA complexes from the immune system. These authors tested several ELR sequences comprising eight VPGXG pentapeptide repeat units, with E/G (hydrophilic and negatively charged), S/G (hydrophilic and neutral), A/G (hydrophobic and neutral) and K/G (hydrophilic and positively charged) as guest residues at the fourth position. The study of IgG and IgM levels in mice after injection of these nanoparticles showed the SG and AG coatings to be the least immunogenic. The presence of the uncharged residues $\mathrm{S}$ and $\mathrm{A}$ changed the conformation of the short recombinamer and allowed the DNA complexes to remain invisible to the immune system. This low immunogenicity (especially for the SG construct) can be explained by the fact that the ELR with serine at the fourth residue produces the most soluble and neutral design among the four constructs. Thus, this study reported several ELRs which could be used to mask drug-delivery systems by avoiding the immune response in mice. This short recombinamer-based strategy to mask the surface of nanoparticles could be useful in the future for producing more efficient and safer gene- and drugdelivery systems, such as nanoparticles, micelles or liposomes [40] .

In another example, Meikle et al. studied the effect of coating superparamagnetic nanoparticles with dendrons in order to control vascular endothelial growth factor (VEGF), which is responsible for angiogenesis in repaired tissue. This growth factor is only effective when a concentration gradient is generated, therefore a new objective could be to develop a system which allows VEGF to be released in the target tissue at desired concentrations. To this end, these authors designed dendrons with a tree-like structure based on lysine monomers to branch the VEGF molecule and four units of the pentapeptide Val-Pro-Gly-Val-Gly. These dendrons are able to bind to superparamagnetic nanoparticles via their root (based on a core made of cysteine and lysine), and these nanoparticles are combined with VEGF by resuspension in a solution of hVEGF. Conformational changes in the dendrons under hyperthermia conditions generated by an oscillating magnetic field leads to release of the growth factor at therapeutic levels. The most important limitation of the application of growth factors in regenerative medicine is the need for local delivery. As patients currently need repeated injections of the growth factor, this design could result in local and targeted delivery of the molecule by generating the concentration gradient needed for correct action of the therapeutic agent [41].

\subsection{ELR-Based nanoparticles as drug-delivery carriers}

In this chapter different ELR-based constructs able to form nanoparticles and their applications as advanced drug delivery systems are covered. From ELRs-diblocks and hybrid diblock ELRs to more complex devices involving triblock copolymers and cell specific ligands are covered.

\subsubsection{Nanoparticles from amphiphilic ELRs}

Amphiphilic ELR-based diblocks are able to selfassemble to form structures such as nanoparticles, thus meaning that, depending on the polymer design, different ways of inducing or reinforcing their self-assembly are possible. Moreover, different bioactive domains have been added to the hydrophilic end so that they are exposed at the outer shell of the particle.

ELRs are characterized by a thermosensitive behavior that can be exploited to perform controlled drug delivery in response to pathological changes in temperature. Local heating at the tumor site is helpful for anticancer therapy with ELR-based systems as they are thermoresponsive and capable of self-assembling into well-defined nanoparticles at the disease site. This hyperthermia, together with the vasodilatation, vascular permeability and increased intracellular traffic into the tumor tissue, will be key factors for cancer treatment [14, 34].

Another stimulus that contributes to cancer treatment is the difference in $\mathrm{pH}$ between normal tissue and the tumor microenvironment. Thus, Callahan and co-workers designed an ELR block copolymer (ELRBC) capable of generating nanoparticles with a $\mathrm{pH}$-responsive moiety, namely [VG7A8]-80/[VH4]-100, that can also be stabilized with $\mathrm{Zn}^{2+}$ ions and which has demonstrated a better in vivo penetration and distribution in tumors than ELRBCs containing non-pH-sensitive ELR block copolymers, [VG7A8]-64/[V]-120. The difference in extracellular $\mathrm{pH}$ between normal tissue $(\mathrm{pH}$ 7.2-7.4) and many solid tumors (pH 6.2-6.9) means that $\mathrm{pH}$-sensitive ELR-based polypeptide micelles disassemble at the lower $\mathrm{pH}$ found in solid tumors. As such, ELRs containing histidine-rich hydrophobic blocks were built, although an evolution of positive charges at the histidine residues at lower $\mathrm{pH}$ was observed as their $\mathrm{pKa}$ is close to 7.4, thus making the ELR more hydrophilic at the tumoral area. $\mathrm{ZnCl}_{2}$ was chosen to stabilize the nanoparticles because is a relatively nontoxic metal ion [42]. The ELRBCs were examined in nude mice bearing HCT-15 human colon adenocarcinoma xerografts. Both polymers maintained the beneficial behavior of high MW drug-delivery nanoparticles, with [VG7A8]-80/[VH4]-100 presenting a lower accumulation in healthy tissues and greater affinity for the lower $\mathrm{pH}$ of the tumor environment. It was also found that [VG7A8]-80/[VH4]-100 shows a more homogeneous spatial distribution and greater penetration within the tumor tissue compared to $\mathrm{pH}$-insensitive ELRBC micelles based on [VG7A8]-64/[V]-120. The histidine-rich ELRBC micelles are able to undergo a pH-triggered disassembly that means the individual polymer chains increase their diffusion capacity and can penetrate inside the tumor. However, $\mathrm{pH}-$ insensitive micelles tend to accumulate around the tumor space as they cannot respond to lower $\mathrm{pH}$ and cannot therefore undergo $\mathrm{pH}$-dependent disassembly, thus meaning that the rate of accumulation in the extravascular space increases. This new $\mathrm{pH}$-responsive mechanism suggests the potential utility of this system for improving drug delivery to, or the imaging of, solid tumors [43].

A new promising method for encapsulation in therapeutic applications involving adding a biomimetic silaffin R5 peptide to the hydrophilic block of the ELR, thus resulting in 
a diblock polypeptide that maintains its ability to selfassemble into micelles and is able to serve as an effective stencil for silicification and formation of nearly monodisperse silica nanoparticles, was proposed by Han et al.. The diblock comprises 60 pentapeptide repeats with valine as a guest residue to provide a hydrophobic block, and 60 pentapeptide residues containing alanine and glycine in a 1:1 ratio as hydrophilic block. Silaffin R5 was then genetically fused to the hydrophilic block of the ELR. This design enables the formation of hybrid organic-inorganic structures that can enhance the stability of hybrid ELR-silica particles and the usefulness of amphiphilic ELRs diblocks by exposing the R5 hydrophilic block at the surface of the nanoparticle because of a high local density of positively charged residues in the silaffin peptide. Silica nanoparticle formation was carried out in phosphate buffer where the ELR-R5 is crosslinked through electrostatic interactions in the presence of phosphate ions, and subsequent addition of silicic acid to the solution of assembled templates provides the silica polycondensation. This method is very attractive for encapsulation of therapeutic agents or imaging moieties in silica-stabilized ELR nanoparticles [44].

Some research has been carried out to improve the NP formation method reported by Van Hest et al., with the strain-promoted azide-alkyne cycloaddition (SPAAC) reaction being used to crosslink the chain at the outer shell of the particle [45]. Thus, a well-defined ELR was designed with the more hydrophilic block fused to a lysine-rich domain to guarantee peripheral crosslinking. The amine groups of the lysines were converted into azide units by a diazo-transfer reaction and, subsequently, the NPs were stabilised by chemical chain crosslinking using biscyclooctyne as reagent. In a parallel study, the NPs were reinforced by covalent interchain crosslinking with genipin, an excellent natural crosslinker for proteins found in gardenia fruit extract [46]. A spontaneous reaction takes place between the lysine residues and genipin, although with longer reaction times than for the SPAAC reaction. In the latter case, the encapsulation ability of the SPAAC crosslinked NPs was investigated by fluorescence emission using a small, labelled hydrophobic molecule. The successful loading of these nanoparticles showed that they may be an interesting candidate as nanocarriers in drug delivery [45].

A new strategy for controlled drug delivery comprises NPs formed from ELR diblocks fused to proteins at the hydrophilic end, which exposes these proteins on the surface for use as a therapeutic agent. This is the case for lacritin, which is a protein with mitogenic and cytoprotective activity that has been studied for the stimulation of wound healing on the ocular surface and for treatment of the corneal epithelium. Thus, lacritin has been fused to the N-terminus of a diblock copolymer ELR to form nanoparticles at physiological temperatures. These nanoparticles were observed by TEM and found to have a mean size of $67.1 \pm$ $11.5 \mathrm{~nm}$ for the structures formed from the fusion protein with lacritin. They were also characterized by optical density measurements and found to have two possible structures, namely stable nanoparticles and the coarcervates formed from them at higher temperatures. The mitogenic activity was confirmed by using SV-40 transduced human corneal epithelial cells, and it was also verified that these nanoparticles can enter the human corneal epithelium using an immortalized human corneal epithelial cell (HCE-T) culture. The results suggest that this system enhances cellular uptake, calcium-mediated signaling, and wound healing. In vivo experiments were carried out with the corneas of non-obese diabetic mice (NOD), and faster wound healing and faster regeneration of the corneal epithelium were observed when nanoparticles were applied topically to the ocular surface. Thus, this study shows the potential of ELR-based nanoparticles as scaffolds for delivering therapeutic proteins to the ocular surface to repair abrasion wounds [47].

A different technique involves the addition of a drug receptor by genetic engineering and the use of polymer nanoparticles as carriers for the drug once joined to its receptor. This alternative encapsulation strategy, which is based on the high specificity between a small molecule drug and its cognate protein target fused to the corona of protein polymer nanoparticles, has been developed by MacKay and co-workers. The drugs are bound tightly to the carrier and are then delivered slowly. Rapamycin (Rapa) is a macrolide antibiotic used to coat stents and prevent organ transplant rejection which also have immunosuppressant functions. Rapa was selected to carry out these assays because of its anti-proliferative properties, low solubility, low oral bioavailability and rapid systemic clearance. Thus, an ELR diblock copolymer containing a hydrophobic block enriched with isoleucine residues and the hydrophilic block with serine residues in a 1:1 ratio for the length of each block, was constructed. Initial experiments had shown that Rapa could not be specifically entrapped in the core of the ELR nanoparticles. Consequently, the cognate protein receptor for the drug (FKBP) was genetically included in the amino acid sequence, thus allowing the drug to be encapsulated into the nanoparticle core via hydrophobic interactions. As a result of this new strategy, the terminal release half-life was increased from $2.2 \mathrm{~h}$ to $57.8 \mathrm{~h}$, with a significant amount of the drug being retained in the core of the nanoparticle and further amounts being bound to the receptor on the surface. This formulation was compared head-to-head with free Rapa in the MDA-MB-468 breast cancer xenograft mouse model. The results showed a high toxicity for free Rapa, with all mice losing more than 15\% of their body weight 23 days after the first treatment. In contrast, the group treated with the nanoparticles showed greater anti-tumor efficacy, showing no signs of behavioral changes or body weight loss, thus indicating a markedly lower cytotoxicity in comparison with free Rapa for that breast cancer xenograft mouse model. This strategy has broader future applications in the encapsulation, targeting and release of other proteins and small molecules [48].

\subsubsection{Nanoparticles from hybrid ELRs}

Different diblock amphiphilic structures generated from ELRs and with both hydrophobic and hydrophilic blocks connected to the ELR biopolymer by chemical bonding or by designing them as a fused protein are discussed in this 
section. These ELR constructs self-assemble into stable nanoparticles when fused to various bioactive blocks such as growth factors or targeting peptides.

A novel method for the formation of nanoparticles as carriers for drug delivery was reported by Nawroth et al., who proposed a hybrid of elastin-like polypeptides conjugated to a poly(2-oxazoline) (POx) block to form a biocompatible diblock polymer precursor for NPs that are typically used in drug, protein, and gene delivery. Moreover, the hydrophilic-hydrophobic balance of this system can be tuned by careful selection of the POx monomer side-chain and the POx-to-ELR molar ratio. The chemical mechanism for POx conjugation is based on a maleimide-thiol click reaction to fuse a hydrophilic cysteine-containing ELR block with free thiol moieties and a hydrophobic POx block carrying maleimide moieties. The resulting structure combines the ability of the POx block to dissolve drugs, good self-assembly properties and low toxicity with the defined nature and biodegradability of ELRs. These conjugates spontaneously form aggregates in water and were used in initial experiments for loading of the anti-cancer drug paclitaxel. Thus, the nanostructures formed were loaded with paclitaxel (PTX) using the thin-film method, in which the drug and the nanoparticles are dissolved in a common volatile solvent, evaporation of which results in the formation of a thin film containing both components. Aggregates incorporating the loaded drug are formed spontaneously upon addition of aqueous solution. The assays realized with ELP-POx at different ELR-to-POx molar ratios showed a clear increase in PTX solubilization as the molar ratio of POx to ELR increased (1:2, 1:4 and 1:8). Indeed, these nanoparticles were able to solubilize the anti-cancer drug with up to $8 \mathrm{wt} \%$ loading [49].

Some research has been devoted to designing ELRs capable of forming nanoparticles that present growth factors on their surfaces and that can interact with cells. These include growth factors for chronic skin wound treatment [50], for the induction of bone regeneration [51] or the treatment of neuronal injuries [52]. Stromal cell-derived growth factor-1 (SDF1) is a small cytokine belonging to the chemokine family that promotes neovascularization and has been proven to provoke a faster re-epithelialization of skin wounds. However, the clinical utility of SDF1 is limited because of its rapid degradation at the wound site due to the high levels of proteases present there. In an attempt to harness the advantages of genetic engineering, SDF1 has been fused to an ELR to produce system that can selfassemble into nanoparticles presenting SDF1 on their surface. Initial in vitro experiments showed that both the fusion protein ELR-SDF1 and recombinant human SDF1 exhibit similar behaviors as regards binding to the CXCR4 receptor and that the biological activity of ELR-SDF1 and free SDF1 is similar and dose dependent in both cases, as measured by intracellular calcium release in HL60 cells. In contrast, the biological activity of SDF1-ELR in in vivo assays using diabetic mouse models was superior to that of free SDF1. Thus, an excisional wound in mice was treated with the same concentration of each and faster wound closure was observed in mice treated with SDF1-ELRs, with
95\% of the wounds being closed after 21 days and fully closed by day 28, while wounds treated with free SDF1 were $80 \%$ closed by day 21 and took 42 days to achieve full closure. These results suggest that SDF1-ELR fusion protein nanoparticles are promising agents for the treatment of chronic skin wounds and a promising method for other wound healing applications, such as myocardial infarction [50].

A similar mechanism has been reported by Johnson and co-workers, who designed two new fused proteins compromising two neurotrophins, namely nerve growth factor (NGF) and brain-derived neurotrophic factor (BDNF). Both NGF and BDN have been shown to improve the outcome of neural injuries in several pre-clinical models, although their clinical use is limited by the lack of a robust delivery system that enhances their bioavailability and halflife. In order to address this problem, NGF and BDNF were separately fused at the $\mathrm{N}$-terminus of the elastin-like recombinamer sequence $\mathrm{V} 40 \mathrm{C} 2$. The resulting constructs ELR-NGF and ELR-BDNF self-assembled into nanoparticles at their respective transition temperatures as a result of the ELR sequence. In order to demonstrate biological activity, in vitro cell cultures were performed using rat PC12 cells to create artificial nervous system tissue models for the study of neurodegeneration. The results showed that the biological activity of NGF was retained in the NGF-ELR fusion construct and enhanced cell survival was achieved by limiting diffusion-related neurotrophin loss. Human epithelial kidney (HEK 293-T) cells transfected with the TrkB-GFP fusion plasmid to express TrkB receptor were used to study the biological activity of the BDNF-ELR fusion protein given the high binding affinity of BDNF for human TrkB. Induction of TrkB receptor phosphorylation suggested that the biological activity of BDNF was also retained in the BDNF-ELR fusion protein. Consequently, the ability to form NPs for use as robust delivery systems, and their enhanced biological activity as fusion proteins, makes these systems promising candidates for use in the treatment of neurodegenerative diseases and as advanced drug-delivery systems [52].

Many ELR-based studies have focused on targeted therapies that are increasingly becoming popular treatments in lung cancer, especially given the highly expressed growth factor receptors found on cancerous cells. As an example, Iglesias et al. designed two chimeric fusion proteins for the delivery of (KLAKLAK)2, a proapoptotic peptide which is responsible for mitochondrial depolarization. The first such chimera is an ELR fused to KLAKLAK, (KLAKLAK)2ELR, and the second one is formed by the same ELR fused to a keratinocyte growth factor (KGF) that is a member of the heparin-binding fibroblast growth factor family (FGF-7), termed KGF-ELR. Cancer cells exhibit higher levels of macropinocytosis than non-cancerous cells, and this can be further enhanced by the presence of growth factors such as KGF due to overexpression of their receptors on those cancer cells expressing high levels of KGF receptor (KGFR). In this study, mixed NPs were formed from two chimeric fusion proteins, namely (KLAKLAK)2-ELR and KGF-ELR. Initial in vitro experiments with human lung carcinoma 
(A549) cells, and (KLAKLAK)2-ELR-based NPs, showed that these nanoparticles were internalized via macropinocytosis and were able to induce cell death, with diffusion of the NPs into the cells facilitated by interaction of the ELR sequence at cell surface sulfate proteoglycans (HSPG). The combination of (KLAKLAK)2-ELR and KGFELR proteins to form mixed NPs has been shown to selectively kill KGFR-expressing lung cancer cells while decreasing the cytotoxicity. These findings demonstrate enhanced macropinocytosis in KGFR-overexpressing cells when using the KGF-ELR fusion protein, thus leading to an increase in (KLAKLAK)2-ELR internalization and subsequent cell death by mitochondrial depolarization. This approach can be used to deliver both peptides and drugs as an intracellular drug-delivery system and thus could be promising as a treatment for lung cancer [53].

Other research in this field has focussed on the genetic incorporation of cell-targeting peptides and internalization ligands along with ELRs. Thus, a new chimeric ELR, known as AP1-ELR-KLAK, comprising the proapoptotic peptide KLAK and multiple copies of an IL-4 receptor targeting peptide (AP1) was designed. This new multivalent drugdelivery system is able to form a structure similar to a nanoparticle at physiological temperature without presenting structural changes or effects on bioactivity, and forms micelles that circulate throughout the body after systemic administration. This multivalent AP1-ELR-KLAK system markedly improved intratumoral localization and retention time compared with the non-targeting control (ELR-KLAK). Moreover, systemic administration of AP1-ELP-KLAK resulted in a significant accumulation thereof in tumor tissue and inhibited tumor growth in various tumor xenograft models by up to $80-85 \%$ by provoking cell apoptosis, with no specific organ toxicity; ELR-KLAK inhibited tumor growth by up to $35-40 \%$. Thus, these new chimeric AP1ELP-KLAK NPs enhanced tumor accumulation and retention time, thus making them promising candidates for effective cancer therapy. Due to the simple preparative procedures of these ELRs, this platform could also be used as a good carrier for tumor-specific delivery of other therapeutic agents [54].

ELRs and cancer therapies are nowadays closely related. As such, numerous researchers have focussed their efforts on developing new strategies that allow a more effective treatment of certain types of cancer. Thus, Janib and coworkers, for example, designed a new fusion protein comprising a recombinant disintegrin named vicrostatin (VCN), specifically designed by Markland laboratory, attached to a high molecular weight ELR called A192. VCN is an antiangiogenic agent with significant anticancer activity, as demonstrated in previous studies, although its major drawback for clinical applications resides in its low molecular weight, which results in rapid renal clearance. To solve this problem, VCN was fused to A192, which has the sequence $\mathrm{G}(\mathrm{VPGAG}) 192 \mathrm{Y}$ and has alanine residues in the $\mathrm{X}$-position, thus resulting in a molecular weight of $80.7 \mathrm{kDa}$, which is well above the renal filtration cut-off. The resulting A192-VCN fusion protein was found to retain its specificity for cell-surface integrins expressed on human umbilical vein endothelial cells (HUVECs). Flow cytometry assays were carried out to determine the specific uptake of ELR-VCN compared with single ELR in HUVECs and in two breast cancer cell lines, namely MDA-MD-435 and MBA-MB-231, with a high and moderate affinity for VCN, respectively, being found. The results showed a nonspecific association in the case of the single ELR used as control protein polymer and a higher binding level in the case of the ELR-VCN fusion protein. Cellular uptake was confirmed by confocal scanning microscopy in HUVEC cells, demonstrating a high and rapid internalization in the case of ELR-VCN. In addition, a saturable tumor accumulation of A192-VCN, which is consistent with integrin-mediated binding, was observed. This receptor-dependent uptake behavior was confirmed by adding an excess of free VCN in order to block integrin-mediated binding, showing almost complete inhibition of the association of ELR-VCN to HUVEC cells and thereby confirming that ELR-VCN is internalized into endothelial cells via heterodimeric integrins targeted by VCN. To determine whetehr ELR stabilization has the potential to shift the biodistribution patterns of VCN, the systemic biodistributions of ELR-VCN, single ELR and VCN were compared using microPET imaging. ELR-VCN was found to display remarkably different cytoplasmatic staining patterns, thus suggesting that the fusion protein promotes the internalization and loss of cell-surface integrin targets. As such, disintegrin fusion proteins may be useful carriers for therapeutic agents or as diagnostic tools [55].

Hydrophobic drugs have also been used as the hydrophobic component of micelle-forming amphiphilic conjugates that can also deliver a payload. In this regard, Zhao et al. have designed a new carrier by conjugating the hydrophobic drug salinomycin (Sali) to a hydrophilic immunotolerant ELR, thus giving rise to an amphiphilic Sali-ELR conjugate that self-assembles into NPs. The resulting carrier can deliver free Sali to solid tumors as a result of enhanced permeability and retention effects. Sali is an antibacterial and coccidiostatic ionophoric therapeutic agent with a selective toxicity for cancer stem cells (CSCs), a subpopulation of cancer cells that have recently been linked with multidrug resistance (MDR) because of their presence in tumors after conventional chemotherapy. Previously reported studies show that charged hydrophobic drugs such as Sali may destabilize NP encapsulation because of their charges. To avoid that effect, free Sali was encapsulated with N,N-dimethylhexylamine (DMHA), which is positively charged. Due to the poor hydrophobicity of DMHA, $\alpha$-tocopherol, a neutrally charged hydrophobic compound, was also used in order to offset the insufficient hydrophobicity. The co-encapsulation of Sali with a combination of DMHA and $\alpha$-tocopherol into Sali-ELR nanoparticles showed an improvement in both the loading and release profile. Moreover, in vivo experiments with Balb/c mice demonstrated an improvement in the pharmacokinetics and a higher tumor accumulation and longer systemic circulation of the drug while also showing that Sali was released from the nanoparticle device at a rapid rate, with a half-life of only one hour [56].

\subsubsection{Advanced strategies for ELR nanoparticle formation}


With the aim of improving controlled drug-delivery strategies, new and more complex devices that take advantage of ELR-based systems to self-assemble to form complex NPs which can carry a wide variety of small molecules have been designed. Self-assembly has been recognized as one of the most powerful techniques for integrating various nanostructured building blocks into macroscopic materials that can translate nanoscale properties into macroscopic devices with novel functionalities.

In this context, Eldijk and co-workers have designed a triblock copolymer named H6-ELR-CP, which includes the capsid proteins from the cowpea chlorotic mottle virus (CP), the self-assembly of which can be controlled by $\mathrm{pH}$, a thermally responsive elastin-like block, and a hexahistidine block that can chelate divalent ions. This copolymer can be used as a supramolecular crosslinker to induce assembly and nanoparticle formation upon addition of these divalent ions. The NP structure can be assembled in the presence of metal ions such as $\mathrm{Ni}^{2+}$ and $\mathrm{Co}^{2+}$, by increasing the temperature and at a $\mathrm{pH}$ of less than 5 , although the NPs dissociate at a $\mathrm{pH}$ higher than 7.5. In order to demonstrate the ability of this metal ion-induced assembly to integrate the drug inside the nanoparticle, a histidine-tagged enhanced green fluorescent protein (H6-GFP) was used. The results of this study showed that encapsulation did not affect the protein shell. In accordance with this, previous studies showed that the internal diameter of the capsules is approximately $5 \mathrm{~nm}$, thus suggesting that they could theoretically contain H6-GFP without affecting the geometry of the capsule. This approach can be extended to other small molecules, peptides or proteins with metal chelating groups for use thereof as nanocarriers for drug-delivery systems [57].

The development of new nanocarriers based on elastinlike recombinamers requires novel strategies. Moreover, modification of these ELRs with cell-specific ligands allows the introduction of active targeting abilities. Thus, Assal et al. designed an ELR fused with a polyaspartic acid tail (ELR-D) and subsequently added epithermal growth factor EGF (ELR-D-E), which will be displayed on the surface of the nanoparticles, to allow active cell-targeting. The resulting nanoparticles were found to present a specific cellular interaction, as revealed by in vitro results with A549 cells, a human lung adenocarcinoma epithelial cell line that overexpresses the EGF receptor.

The same group has also designed an advanced approach for loading a single-chain vascular endothelial growth factor (scVEGF121)-derived polypeptide into the nanoparticles. With the aim of providing noncovalent tethering upon formation of a heterodimer coiled-coil structure, two peptides known to establish this structural motif, one containing basic (helixB) and the other acid (helixA) residues, were fused to the previous ELR-D and to scVEGF121, respectively. The ELR-D block combined with helixB at its C-terminus to form nanoparticles that respond to temperature changes and are able to encapsulate small molecules and drugs above their phase-transition temperature. When combined with the block scVEGF121, HelixA exhibits a tumor-targeted ability, thus indicating that this system is able to carry the drug to its destination. To confirm the efficacy of this novel strategy, assays involving exposing the growth factor on the surface of nanoparticles loaded with the anticancer drug PTX to target the VEGF receptor of cancer cells were carried out. This VEGFtethered ELR-D-helixB construct was found to exhibit enhanced cytotoxicity against HeLa cells compared with ELRD-helixB, with marked cell death being observed after only 6 hours post-treatment and almost complete cell death by 12 hours, similar to a positive control with free PTX. This nanostructure reduces renal clearance with respect to free PTX and allows a more specific delivery. Furthermore, the combination of coiled-coil heterodimers that are able to form nanoparticles with targeted drug delivery enhances tumor suppression and increases apoptotic cell death. This study demonstrates that customized drug-delivery systems that target different cell types and receptors can be constructed using ELRs and may be a good candidate for cancer therapy applications [58].

The literature contains numerous examples of nanoencapsulation methods for advanced drug release, and specifically for cancer treatment. Indeed, the design created by $\mathrm{Hu}$ et al. is remarkable in this regard. These authors reported a general platform for precisely designing tumorhoming and $\mathrm{pH}$-responsive ELR-drug nanoparticles for targeted cancer therapy. Thus, the ELR designed, which comprises three blocks, is known as F3-ELR-C8. The first block (F3) is a 31 amino acid sequence from the $\mathrm{N}$-terminal fragment of human group protein 2 (HMGN2), which specifically binds to the nucleolin expressed at the membrane of tumor endothelial cells and tumor cells. The Cys-rich block (C8) is genetically fused to the biopolymer at its C-terminus and is the site selected for the conjugation of drugs such as doxorubicin (Dox) via an acid-labile hydrazone linker. This resulted in the formation of a triblock (F3-ELR-C8-DOX) loaded with a therapeutic agent that can self-assemble into tumor-targeting and $\mathrm{pH}$-responsive polypetide-drug nanoparticles in aqueous solution (Figure 1). Pharmacokinetics assays were carried out with a murine cancer model by dialysis against PBS at $\mathrm{pH} 7.4$ and $\mathrm{pH} 5.5$ at a temperature of $37^{\circ} \mathrm{C}$. Less than $8 \%$ of the drug was found to be released at $\mathrm{pH} 7.4$ over $24 \mathrm{~h}$, whereas nearly $50 \%$ of the drug was released at pH 5.5 after only $6 \mathrm{~h}$, thus indicating the stability of the hydrazone bond at blood $\mathrm{pH}$. In vivo assays in a murine cancer model showed that F3-ELRC8-DOX nanoparticles resulted in 4.2- and 1.8-fold higher drug concentration in the tumor compared with free DOX and ELR-C8-DOX, respectively, thus indicating an enhanced anti-tumor efficacy and a significantly reduced cardiac toxicity, both of which are highly desirable features for the development of advanced drug-delivery systems for targeted cancer therapies [59]. 
use in tissue regeneration, neuroinflammation, cancer and diabetes. The genetic design of these ELRs allows their characteristic $\mathrm{Tt}$ to be controlled below the physiological temperature of $37^{\circ} \mathrm{C}$, thus resulting in situ depot formation and maintaining adequate therapeutic levels at the desired site while avoiding systemic exposure [17]. Moreover, the polymer chains can be interconnected by covalent or not covalent crosslinking, thus stabilizing the structure and allowing the formation of hydrogels that can be used as injectable biomaterials.

\subsubsection{In situ Therapeutic ELR Depots}

Anticancer therapy is an example of a treatment in which prolonged local exposure of the tumor is crucial to its success. Most systemic administrations result in high toxicity rates [61] and, moreover, therapeutic agents must pass through several biological barriers to reach the tumor and ensure an effective dose [17]. Fortunately, these drawbacks can be avoided by intratumoral administration, which facilitates localized anticancer drug exposure and maximizes drug retention within the tumor. The development of a biomaterial that can be injected at room temperature as a drug-delivery depot provides an attractive alternative for local delivery. In this case, the thermoresponsive ELR sequence was designed to provide a biopolymer which was injected into a tumor when soluble and formed a depot in situ due to temperature-triggered coacervation. The thermoresponsive ability of ELRs makes them a suitable alternative as anticancer treatments in combination with anticancer therapeutic agents such as iodine-131 radionuclide. In this regard, Chilkoti's group has developed two different labelled ELRs, one of which is soluble (with $\mathrm{Tt}>60{ }^{\circ} \mathrm{C}$ to ensure solubility at body temperature) and the other

which

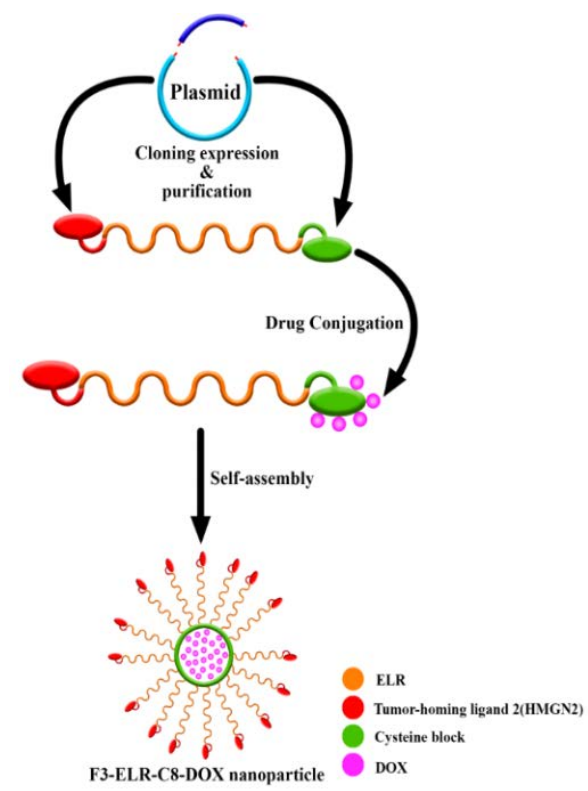

has a give positively charged and stable polyplexes under physiological conditions. The incorporation of MUC1specific aptamers into the polyplexes gave rise to NP constructs with positively charged surfaces and with an adequate particle size for cell internalization, as well as cellline specificity provided by the aptamer. This system was studied as a candidate for application in suicide gene delivery using a plasmid containing the gene for the toxin PAP-S and was found to deliver the plasmid into tumor cells, with increased specificity for MCF-7 breast cancer cells with respect to a MUC-1-negative tumor line, while protecting normal human cells. The transfection ability of this aptamerELR vector, as determined by micropinocytosis uptake, shows its potential application for suicide gene therapy in cancer treatments and continues to pave the way for the application of ELRs in the biomedical field [60].

\subsection{Macroscopic ELRs for drug delivery}

ELRs are currently being widely used for the development of novel drug-delivery devices that allow local exposure of the treatment. The thermoresponsiveness of ELRs makes them an interesting biomaterial for the development of biomedical devices, such as drug devices for

thermoresponsive ability (Tt of $28^{\circ} \mathrm{C}$ by incorporation of $\mathrm{Val}$ at all guest residue positions). In vivo results showed that ${ }^{131}$ I-labeled ELR depots produced higher tumor regression values and survival rates compared with a soluble ${ }^{131} \mathrm{I}-$ labeled ELR control, although complete regression could not 


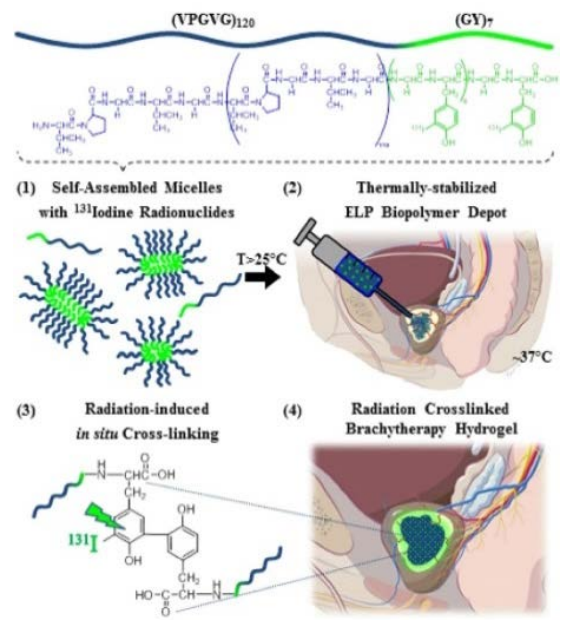

be achieved using this strategy [62]. As a result, these labelled ELRs were improved [63], with their design being optimized by introducing larger elastin domains and tyrosine-enriched domains. Modification of the injection concentration also improved the strategy. In this case, higher tumor retentions and $67 \%$ complete regression were obtained after 60 days in two xenograft mouse models of $\mathrm{FaDu}$ squamous cell carcinoma and PC-3 prostate cancer.

Intratumoral radiation therapy (brachytherapy) is a highly successful treatment for solid tumors, particularly cancerous prostate ones. In this technique, radioactive "seeds" are implanted in or near the tumor location, thus providing a high radiation dose to the tumor while reducing unwanted exposure in the surrounding healthy tissues [64]. Despite the overall benefit of this technique, current seed implants are permanent and their beneficial results are limited in clinical application to malignancies of low or intermediate grade, with their use not being possible in high grade cases. Numerous attempts have been made to develop polymeric devices in combination with brachytherapy [65-66]. In order to avoid the problems associated with conventional brachytherapy [67], Schaal et al. developed an injectable polymeric approach for delivering brachytherapy that is able to self-assemble in situ. The previously mentioned ${ }^{131} \mathrm{I}$ labelled ELR was used in this case in the form of micelles. The choice of micelles rather than unimers was due to the ELR micelle-coacervate transition, which is independent of injection concentration and is therefore a key factor in the ability of ELR unimers to coacervate. This conjugated biomaterial was stabilized in two different ways: firstly, body temperature was used to trigger a rapid phase transition (less than 2 minutes) in the micelles to form an insoluble, physically stabilized coacervate; secondly, the high energy $\beta$-emissions of ${ }^{131}$ I further stabilized the depot by tyrosinemediated crosslinking via a radiation-induced reaction within the ELR depot over the course of $24 \mathrm{~h}$ (Figure 2). The ELR depots were evaluated in a prostate and a pancreatic mouse model, achieving tumor regression values of around $95 \%$ in the prostate tumor model and a sixfold higher median survival (from 12 days to more than 60 days) with respect to the control group. For the pancreatic tumors, ELR brachytherapy significantly increased growth inhibition and enhanced median survival to 27 days compared with a median survival of 8 days shown by controls [68].
Figure 2. Design and mechanism of ELR-based brachytherapeutic depot formation. (1) Initial self-assembly of the micelles with ${ }^{131}$ I-radionucleides; (2) ELR depot formation stabilized by a fast phase transition; and (3) Radiation-induced cross-linking reaction mediated by high energy $\beta$-emissions of ${ }^{131}$ I produces chemical crosslinkages within the ELR depot (4), thus resulting in a stabilized hydrogel. Reproduced with permission from Schaal et al. [68].

Over the past few years, ELR-based devices have been considered for use in topical ocular administration, especially as regards the development of new release mechanisms given the high incidence of ocular-related diseases [69-70]. Lacritin (Lacrt) is closely related to a group of novel factors that are able to promote tear protein secretion and stimulate mitogen corneal activity [71]. As such, Lacrt was fused to an ELR comprising 96 repetitions of the pentapeptide VPGVG to give a monomer that is able to form an intra-lacrimal depot at physiological temperature, thus allowing excellent control of drug localization and maintaining Lacrt-mediated cell-signaling pathways. The Lacrt-ELR construct was able to enhance $\beta$-hexosaminidase secretion and actin remodeling, as shown in in vitro studies in isolated primary cultured lacrimal gland acinar cells. Likewise, in vivo results showed an enhancement in tear secretion in both male and female NOD mice (around $40.9 \%$ and $50.9 \%$ respectively) compared with the ELR or free Lacrt alone, which had an effectiveness of $29.6 \%$ in males and $42.9 \%$ in females [72].

Although cytokine agonist-based therapies are an attractive option for the treatment of post-traumatic arthritis (PTA), intra-articular injections of anti-cytokine therapies have encountered difficulties owing to rapid clearance from the joint space, and systemic administration is limited by the lack of vasculature. In light of this, Kimmerling et al. have developed a novel cross-linked ELR-based (xELR) spongelike drug depot that is able to encapsulate IL1Ra (IL1 receptor antagonist) and TNFRII (TNF $\alpha$ inhibitor) for intraarticular delivery thereof [73]. The therapeutic efficacy of the prolonged delivery of IL-1a and/or TNF- $\alpha$ inhibitor was evaluated in a murine intraarticular defect model. These in vivo studies showed that inhibition of IL-1 significantly reduces the severity of cartilage degeneration, synovial inflammation and facilitates bone healing, all of which that are closely related to PTA. Conversely, inhibition of TNF- $\alpha$ alone, or with IL1Ra, results in deleterious effects on bone morphology and articular cartilage degeneration owing to the inhibition of TNF- $\alpha$. This novel device comprising a combination of xELR with IL-1a for sustained intra-articular delivery allows a marked decrease in clearance from the joint space, thereby improving the effects of IL-1a and minimizing the dose and frequency of the injection [74].

\subsubsection{ELRs depots as Macromolecular Carriers}

It has been demonstrated that ELR depots maintain their therapeutic effect even when not present at the site of the disease, thus providing a long-circulating macromolecular carrier for effective peptide-based drug treatments. Thus, a fused ELR depot was used to deliver glucagon-like peptide-1 
(GLP-1), an endogenous increin secreted by intestinal cells in response to food intake, in order to promote insulin exocytosis in a glucose-dependent manner [75]. This peptide-based drug has demonstrated great promise for the treatment of type 2 diabetes, although its relatively low halflife due to plasma clearance and rapid enzymatic degradation highlights the need to improve this strategy. In order to improve the results obtained with this type 2 diabetes treatment, Chilkoti and co-workers developed a novel strategy based on the glucagon, which was introduced into the organism in a depot form [76]. In vivo results in a mouse model susceptible to developing type 2 diabetes revealed that a single injection of the GLP-1 ELR depot was able to reduce glucose levels by $30 \%$ for 5 days. Moreover, its efficiency was five- and 120-times better than the free GLP1 and GLP-1 ELR fusions that did not aggregate at body temperature, for which no rapid decrease in glucose levels or any sustained release were shown. The GLP-1 ELR depot maintained its therapeutic activity, maintaining adequate glucose levels and resulting in prolonged release, as a result of the ability of the ELRs to coacervate. Alternatively, the aforementioned fused GLP-1 ELR was genetically modified by introducing flanked protease cleavage sites, thus giving rise to a Protease Operated Depot (POD) that is able to release the free GLP-1. Remarkably, a single injection of a POD containing six GLP-1 repeats on each ELR demonstrated a reduction in blood glucose levels in mice for up to 5 days, six times longer than with an injection of the soluble GLP-1 ELR control and 120 times longer than the free peptide drug ( $6 \mathrm{~h}$ and $1 \mathrm{~h}$, respectively). These findings demonstrated that PODs provide an innovative alternative to synthetic peptide encapsulation devices for the sustained delivery of peptide therapeutics.

ELR depots can also play an important role in other disease states, such as neuroinflammation, in which local treatment is recommended order to improve the antiinflammatory therapeutic effects and avoid harmful side effects. In this regard, tritium-labeled ELR depots at the perineural site were evaluated, resulting in a sevenfold increase in half-life in comparison with the soluble ELR under physiological conditions while minimizing systemic exposure, thus proving therapeutically effective [77]. Another interesting strategy in the development of neuroinflammation therapies is the use of ELRs covalently conjugated to small molecule drugs such as curcumin. Thus, this small TNFa antagonist was conjugated to ELRs containing periodic reactive glutamate residues via a degradable carbamate linker [78]. In vitro results showed that the conjugated ELR depot maintained its ability to coacervate and the TNF $\alpha$ inhibitory activity of curcumin was largely conserved compared to the free drug. Conjugated depots injected intramuscularly close to the sciatic nerve retained fivefold higher levels of curcumin at four days than for the free drug, and systemic curcumin exposure was also reduced sevenfold in plasma.

The combination of thermally responsive ELRs with different strategies can thus offer a wide variety of mechanisms related to drug delivery as either depots for prolonged in situ release of peptide ELR fusions or as depots that provide a carrier for free peptide drug delivery, thereby sustaining their therapeutic effects when compared with the free drug.

\subsubsection{Hydrogels from ELRs}

Hydrogels are a class of highly hydrated polymers composed of a network of insoluble three-dimensional, hydrophilic polymer chains that are able to swell in water but do not dissolve. Such networks can be formed via crosslinking in chemical reactions or by physical complexes triggered by different stimuli such as $\mathrm{pH}$, temperature, or ultrasound energy [79]. In general, the structural integrity of such hydrogels depends on cross-linking or physical entanglement between polymer chains [80]. Hydrogels are able to circumvent some delivery disadvantages due to their ability to be placed near the site of interest and deliver drugs locally over a prolonged period of time. This local exposure at the target site helps to maximize the time during which systemic effects can be minimized.

A novel ultrasound method has been used to prepare a physical ELR hydrogel scaffold for local delivery of the model protein BSA (bovine serum albumin) and an antibiotic (doxycycline hyclate) commonly used in periodontal therapy. These hydrogels are formed by sonication for a short period of time in aqueous solution on ice, thus ensuring that the solution temperature remains below Tt. Further incubation of the ELR at $37^{\circ} \mathrm{C}$ in the presence of bovine serum albumin and doxycycline hyclate, which were added to the sonicated ELR, provides a hydrogel with the therapeutic agents enclosed. The release of BSA and doxycycline from the scaffold was studied at different temperatures above $\left(37^{\circ} \mathrm{C}\right)$ and below $\left(25^{\circ} \mathrm{C}\right)$ the transition temperature $(\mathrm{Tt})$, and with different loading concentrations of the two bioactive molecules. A comparison of the release profiles of doxycycline and BSA showed that release was only higher at $25{ }^{\circ} \mathrm{C}$ than at $37{ }^{\circ} \mathrm{C}$ for the higher concentration of BSA $(1 \% \mathrm{v} / \mathrm{v})$ studied. These results suggest that the molecular weight of the drug and loading concentration affect the release kinetics. The highlight of this approach is the ease of formation of ELR hydrogels without the need for any sophisticated instrumentation (such as electrospinning), chemical agents for crosslinking, and/or organic solvents. Moreover, the release profile was supported by different factors such as temperature or drugloading dose, thus making it ideal in controlled drug delivery and cell culture applications [81].

One of the most innovative hydrogel-based devices for drug delivery comprises enzymatically cross-linked ELRs that are able to release bioactive agents under the effects of a specific environmental stimulus. As described previously [82], there is a correlation between elastolytic activity and pathological conditions such as atherosclerosis, cystic fibrosis or chronic wounds, amongst others. In this regard, Bandiera et al. have developed an ELR hydrogel matrix containing an enzymatic cross-linking domain susceptible to proteolytic degradation. Crosslinking was achieved by addition of microbial transglutaminases and the resultant hydrogels were exposed to the proteolytic activity derived from P. aeruginosa and human polymorphonuclear 
leukocytes (PMNs), thus leading to sustained release of the recombinant green fluorescent protein (eGFP) embedded during the gelation process. Similar results were obtained upon embedding cytochrome $\mathrm{C}$ within the matrix, although the significant interaction observed between the matrix and the drug delayed release from $10 \mathrm{~h}$ to $50 \mathrm{~h}$. In summary, a drug-delivery device that is able to respond to environmental changes for therapeutic agent release has been designed [83]. Moreover, this eGFP delivery device could represent a good model for the most appealing possible applications, for example the controlled release of bioactive factors such as growth factors, hormones, antibodies, and other therapeutic macromolecules.

The multiresponsive ability of ELRs makes them a suitable biomaterial for producing physically cross-linked depots or hydrogels under specific conditions. However, noncovalent cross-linking usually results in poor structural stability and mechanical properties, thus making them unsuitable for some applications. In order to achieve better results in the applicability of these biomaterials, Chilkoti's group has developed an injectable, reversible, disulfidemediated cross-linked hydrogel, with properties intermediate between those of coacervates and more robust chemical hydrogels, by engineering the presence of cysteine residues along the ELR structure [84]. These engineered hydrogels undergo rapid gelation under the mild oxidative conditions provided by addition of a low and physiologically appropriate concentration of hydrogen peroxide (0.3 wt. \%). Preliminary in vitro studies on the release profiles of BSA as model protein have shown that the cELR hydrogel exhibits first-order kinetics for release of the entrapped BSA, which starts with an initial burst followed by a prolonged gradual release (20-40\%) after 4 hours of incubation compared with the complete release observed for the control gel (ELR with no cysteine or induced cross-linking). Furthermore, a potential use of these disulfide cross-linked cELR hydrogels for the local delivery of radionuclides within tumors has been evaluated. Thus, the in vivo intratumoral injection of radiolabelled cELR to nude mice bearing human pharynx squamous xenografts showed enhanced tumor retention (greater than $40 \%$ of the payload) in addition to a homogeneous spatial coverage across the entire tumor as compared to the above-mentioned soluble ELR control. These results show the utility of these hydrogels for the sustained release of a model protein in vitro, and demonstrate the ability of this injectable biomaterial to serve as a delivery system.

SELRs are chimeric recombinant polymers whose sequence contains selected domains from the structural proteins silk and elastin. As such, they combine specific properties of both native proteins, thereby displaying unique mechanical properties that combine the high strength of silk with the resilience of elastin while incorporating its multiresponsive sensitivity. This versatile behavior provided by the silk and elastin blocks makes SELRs a promising biomaterial of application as injectable drug-release systems [8587].

Colino-Fernandez et al in order to find new solutions and approaches to overcome the current problems of ophthalmic drug delivery have developed a new antiglaucoma formulation in order to reduce intra ocular pressure (IOP), one of its main risk factors. Two different ELRs, one containing the silk domain (SELR), have been used to release timolol maleate (TM), the most used treatment for IOP. In vitro test shown clear differences in the drug-release profiles, the SELR system shows a more sustained release when compared to its ELR, with the percentage release after $8 \mathrm{~h}$ being $40.04 \%$ and $80.39 \%$ TM, respectively. These data point to a relationship between the more enhanced mechanical properties of SELR due to the stability provided by the silk domain and the rate of drug release. These results were corroborated by in vivo studies carried out in normotensive male New Zealand white rabbits. While those formulations containing $\mathrm{TM}$ and the respective recombinamer maintained a reduced IOP after 4h, the TM solution presented no hypotensive effects, being more pronounced for the formulation containing SELR. In this case, the hypotensive effect lasted for more than $8 \mathrm{~h}$, which is in agreement with the enhanced stability of this material. Furthermore, these recombinamers can be placed in the eye without causing irritating effects or tear turnover, as demonstrated by the irritation tests, thereby maximizing the potential utility of these devices as drug-delivery systems. Therefore, SELRs constitute a novel and versatile type of hydrogel to address the critical issues that ophthalmic drug delivery entails [88].

Based on a previous study, Price et al. modified an SELR by introducing a lysine in position 815 (SELR815K) of the elastin block along with longer silk and elastin domains, thereby enhancing the ability for local delivery of adenoviruses [89] and gaining further hydrogel robustness as a result of the silk domain. With the aim of developing more resorbable biomaterials, this SELR815K was combined with a matrix metalloproteinase (MMP)-responsive sequence at three different block positions (Figure 3). In vitro results revealed that the degradability of the SELR was affected by the presence of the MMP-responsive sequence [90] in the presence of MMP-2. Moreover, the position of the MMPresponsive domain in the silk block was determinant for the degradation rates, resulting in a reduction of $20 \%$ when the silk domain was altered. These results were corroborated by in vivo experiments in a murine head and neck tumor model. The construct in which the elastin or silk domains were not affected was found to be the most effective cancer treatment for the delivery of viral gene therapy, increasing mice survival from $29 \%$ to $100 \%$ compared with the control group.

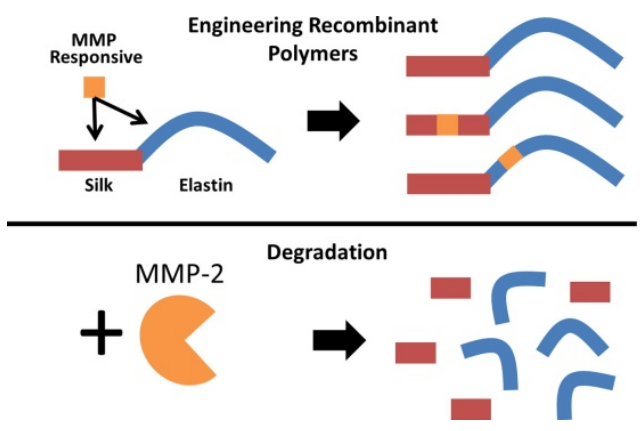




\section{TISSUE ENGINEERING AND REGENERATIVE MEDICINE}

Tissue engineering and regenerative medicine are very useful tools for restoring, maintaining, or improving viable biological tissues or whole organs after a loss of their functionality [97]. Over the past few years, materials science for tissue regeneration has evolved from the production of a support that is "tolerated" upon direct application to a damaged area but is not able to self-regenerate to the development of advanced devices that mimic physiological environments and are able to interact with the body. Indeed these interdisciplinary fields of biomaterials science are slowly combining the use of innovative scaffolds, suitable cells and bioactive molecules to regenerate functional tissues [98]. Several issues have been considered to be essential for the construction of an effective scaffold, namely i) the threedimensional nano-microstructure required, ii) its porosity, iii) its specific mechanical characteristics, iv) its wettability and permeability, v) the need for manipulation and the discomfort caused to the patient, vi) cell-material communication via biochemical signals, and vii) the time of residence before host tissue remodeling [99]. Taking advantage of their recombinant nature, biocompatibility and mechanical features, elastin-like scaffolds have been designed and produced to reproduce some of the properties of the ECM such as architectural topographies, suitable firmness and porosity, promotion of cell adhesion and viability, cell signaling responsiveness, biodegradability and stimuli-responsiveness to changes in their environment, amongst others [100-104]. In this review we describe some different approaches in which elastin has been engineered to obtain customized supports adapted to the part of the body to be treated, such as the skeletal system [105-108] and vascular system [109-110], and also for the regeneration of soft tissues, with some of the most recent examples being discussed below.

showed a slower release of doxycycline compared to collagen hydrogels, probably due to a smaller swelling ratio as a result of secondary bonding interactions between the components of the composites. In order to test the bioactivity of doxycycline released from the hydrogels, bioassays against four bacterial strains commonly encountered in clinical settings (E. coli, P. aeruginosa, S. sanguinis and methicillin-resistant Staphylococcus aureus) were performed using a disk-diffusion method. The results obtained revealed that the doxycycline released from all hydrogels was effective against all four bacterial strains tested, thus confirming the absence of any interference of the hydrogels with the bioactivity of the drug. As such, combined ELRcollagen hydrogels allow the sustained release of a model antibiotic at the local site and could therefore potentially be used to reduce post-surgical infections and facilitate wound healing.

\subsection{Skeletal System regeneration}

The human skeletal system serves as a framework for the body and consists mainly of individual bones and cartilages. Bone is a tissue that possesses an intrinsic capacity to regenerate in response to continuous remodeling stimuli throughout adult life or as a result of injury. Despite this regeneration ability, self-healing conditions that are detrimental to the process, such as complex skeletal defects due to the size of the fracture or neoplastic or infectious bone disease, can be limiting [111-112]. In these cases, tissueengineered supports are required to ensure structural functionality and to drive bone reconstruction as a result of their osteoconductive, osteoinductive, and osteogenic components (Figure 4). 
Figure 4. Schematic representation of bone tissue hierarchically organization: the macroscopic level (a), the osteon the osteon units and the collagen fibers concentric layers "lamellae", nerves and vasculature (b) the molecular structure of collagen organized in fibrils that include hydroxyapatite crystals for increasing the rigidity of the bone (c). From (d) to (i) are shown different nanostructural features (patterns, fibers, pores or composites) of bone substitutes prepared to mimic bone structure. Reproduced with permission from Gong et al [112].

In their search for this kind of innovative support for bone regeneration, Coletta et al. developed two different ELRs that form thermoresponsive self-assembled hydrogels under physiological conditions due to their amphiphilic composition. Moreover, the sequences of these ELRs contain multiple bioactive motifs, such as the osteogenic and osteoinductive bone morphogenetic protein-2 (BMP-2) or domains from human fibronectin containing the Arg-GlyAsp (RGD) motif in order to promote integrin-mediated cell adhesion, in addition to elastase-sensitive sequences to study biodegradation of the ELR molecules. The BMP-2 component stimulated osteoblast proliferation, whereas the RGD sequence helped host cells to migrate inside the scaffold and increase cell viability. Interestingly, this work showed how biodegradation of the scaffold occurred concurrently with bone regeneration, thus confirming that host bone remodeling determines degradation of the temporary scaffold. An in vivo study in female New Zealand white rabbits demonstrated that these showed hematopoietic bone marrow and the invasion and proliferation of osteoblast layers, thus suggesting a high potential for the repair of bone defects and excellent biocompatibility [113].

In a previous study, to enrich the osteogenic and osteoinductive properties of ELR scaffold, the recombinant human BMP-2 was included in a fusion protein containing an ELR block comprising pentapeptides including valine and cysteine, known as V40C2, which self-assembles at $37{ }^{\circ} \mathrm{C}$. The main aim was to determine whether the fusion protein retained the bioactivity of BMP-2. The comparison between fusion protein and the commercially available recombinant BMP-2 showed that both retained bioactivity even is was reduced in the ELR fusion protein. This reduction could be attributable to its partial degradation observed during its production. When compared with the control, BMP-V40C2 showed higher alkaline phosphatase activity, higher mRNA levels of osteoblast-specific transcription factor Osterix (OSX) and higher calcium deposition after a 21-day treatment. As such, this study demonstrated that the fusion protein not altered the osteogenic ability of BMP-2 and that the construct retained the transition ability of ELRs [114].

Another interesting strategy involves combining siliconsubstituted hydroxyapatite (Si-HA) scaffolds and ELRs to produce bone tissue substitutes. HA is a major component of human bone and teeth and is frequently used in orthopedic, dental and maxillofacial applications owing to its biocompatibility, osteoconductivity and bioactivity, which mean that it supports bone growth and osteointegration. In

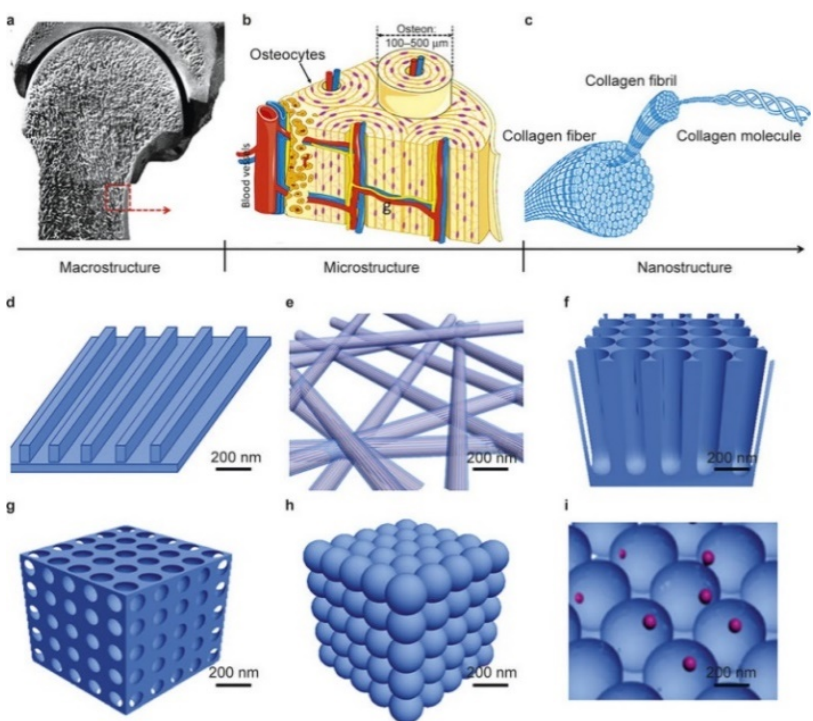

this study, the authors used 3D bio-resorbable scaffolds comprising ceramic Si-HA with controlled porosity, the surface of which was functionalized with two bioactive ELRs containing the cell adhesion peptide RGD, either alone or together with the $\mathrm{N}$-terminal residue from statherin (SNA15 peptide), which has shown a high affinity for calcium phosphate minerals such as HA and the ability to mediate cellular function at the mineral interface, thereby facilitating the mineralization process. Strikingly, in vitro studies resulted in the same viability and growth of rat bone marrow mesenchymal stromal cells (rBMSCs) on Si-HA, SiHA-RGD and Si-HA-SNA15/RGD. Moreover, there were no significant differences between the colonization rate of cells in the 3D scaffolds. However, when osteoblast differentiation was evaluated, expression of the osteoblast markers OC and ALP demonstrated that the Si-HA-SNA15containing scaffolds were very efficient at leading the rBMSCs into the osteoblastic lineage. In summary, we can conclude that although cells were able to grow on and colonize any of the scaffolds tested, their behavior differed and the biochemical signal generated by the functionalized ELR significantly enhanced the osteoconductive properties of the scaffolds [115]. In contrast to bone, some tissues, such as enamel for example, are unable to regenerate. As such, Shuturminska et al. have developed an ELR containing the $\mathrm{N}$-terminal sequence of statherin, a small protein which helps enamel remineralization. When the resulting STNA15ELR was incubated in a mineralization solution at $37^{\circ} \mathrm{C}$, mineral platelets appeared after 8 days, whereas they did not appear in the uncoated substrate. Thus, ELRs could have an important role for stabilizing the mineral. SEM images confirmed the presence of a mixture of calcium fluoride and fluorapatite at day 8. Moreover, TEM images also showed the presence of fluorapatite. In summary, this preliminary work could lead to the development of a promising material for enamel biomineralization [116].

An ability to achieve the optimal biomechanical properties for the development of skeletal-scaffolds to use in tissue engineering has been one of the most difficult challenges for researchers for a number of years. For this reason, several studies have been carried out to improve the 
strength of ELR-based scaffolds. In this regard, the inclusion of cysteine residues and the RGD peptide resulted in a cytocompatible hydrogel exhibiting long-term resistance to erosion. Indeed, mass loss was demonstrated to occur as a result of contraction rather than erosion in this system, and human mesenchymal stem cells (hMSCs) cultured on the hydrogels seemed to be remain viable after 21 days. Moreover, these hMSCs showed higher levels of osteogenesis markers, such as osteocalcin and osteopontin, when compared to hMSCs plated on glass slides. Alkaline phosphatase activity was also higher with respect to the control, and chondrocytes added to the gel exhibited $86 \%$ viability and good morphology after culture for 28 days [117].

As we explained in the introduction, one of the more interesting applications of elastin-like biomaterials is their ability to create biomimetic scaffolds. As 2D cultures do not reproduce the physiological 3D environment, Gurumurthy et al. studied 3D spheroid culture with an ELR chemically conjugated to polyethyleneimine (PEI) and compared the resulting construct with a 2D monolayer culture. Thus, these authors seeded human adipose derived stem cells (hASCs) on standard tissue culture polystyrene coated with the positive charged ELR-PEI, thereby forming 3D spheroids as a 3D culture model. After incubating both in differentiation media, viability assays were performed and showed the same percentage of live cells as for the 2D monolayer. This result confirms the lack of cytocompatibility problems for the 3D spheroids. Furthermore, this spheroid model presented significantly higher ALP activity and osteocalcin production. Mineralization was also higher for the 3D spheroids when compared to the 2D model. In conclusion, 3D spheroids were found to be a better system for hASC differentiation with respect to the standard culture methods [118].

The bicomponent supports, ELR-collagen scaffolds, whose advantages have been previously described were also developed as composites to guide bone regeneration. ELRs that provide strength and extensibility can enhance the mechanical properties of the scaffolds, and to study that four different composites with increasing ELR/collagen ratios have been compared. Despite the fact that all these composites exhibited hASC proliferation and differentiation, the composite with the highest ELR/collagen concentration $(18 / 6 \mathrm{mg} / \mathrm{mL})$ exhibited the highest ALP activity, OCN expression and mineralized matrix. Collagen-only composites and those with lower ELR/collagen concentrations showed slower maturation and a less mineralized matrix. As such, the incorporation of ELR and a reduction in the collagen content to $25 \%$ resulted in the best scaffold for osteogenic differentiation of hASCs. In addition, this scaffold may be able to minimize collagen degradation in future in vivo assays [119].

The degeneration of joint cartilage is currently one of the most common causes of discomfort and disability in the elderly. However, the self-regeneration of joint cartilage damaged as a result of injury or degenerative joint disease is especially difficult to achieve due to the low intrinsic capacity of cartilage for self-renewal given its avascular nature [120]. As hyaluronic acid (HA) is the major component of cartilage extracellular matrix, it is an interesting material for studying cartilage regeneration. Similarly, hydrogels are attractive scaffolds because of their mechanical properties and their ease of manipulation, especially those that can undergo self-gelation under physiological conditions and could therefore form a device in situ. As such, ELR-HA hydrogel scaffolds containing encapsulated chondrocytes have been proposed and a comparative study of scaffolds with different component ratios has been carried out. The stiffness of the supports was unchanged in the range of ratios evaluated. As the increased proportion of HA was increased, gene expression of cartilage markers in chondrocytes, such as aggrecan, SOX9 and type 2 collagen, increased in a dose-dependent manner. The importance of these chondrocyte markers should be highlighted as chondrocytes are the only cells present in cartilage and they are involved in maintenance of the extracellular matrix. Moreover, when the HA concentration was decreased, the chondrocytes encapsulated in the hydrogel showed upregulated levels of type 1 collagen (characteristic of an undesired fibrocartilage) and metallopeptidase-13, thereby suggesting matrix degradation and remodeling. In addition, hydrogels with a lower HA concentration exhibited more chondrocyte proliferation, whereas higher HA contents resulted in decreased cell proliferation and more cartilage-specific matrix sGAG (sulfated-glycosaminoglycan) deposition. This system was therefore identified as the best scaffold candidate (5\% HA hydrogel) as it exhibited the highest expression of cartilage markers, more sGAG deposition, less cell proliferation and less production of type 1 collagen and fibrocartilage phenotype [121].

\subsection{Cardiovascular system regeneration}

Vascularization control is one of the main challenges in tissue repair. In this regard, artificial tissue engineering scaffolds have to allow the correct flow of oxygen and nutrient and the removal of catabolism products. The formation of an efficient network of blood vessels and capillaries in the scaffold is therefore necessary [122]. Although numerous different biomaterials available, ELR hydrogels provide a viscoelastic, biocompatible and bioactive material [123]. Staubli et al. tested in vitro vascularization by encapsulating human adipose tissuederived SVF cells into different ELR hydrogels, ranging from non-functionalized hydrogels (NF-hydrogels) to an RGD/REDV-containing hydrogel with two cell adhesion integrin-mediated motifs (REDV is specific for endothelial cells). The SEM images obtained confirmed that the nonfunctionalized system showed low cell adhesion and that the RGD/REDV hydrogel exhibited well-organized flat, elongated parallel cells. Furthermore, CD-31 staining showed that endothelial cells formed aggregates in the RGD/REDV hydrogel but not in the non-functionalized hydrogel. Interestingly, cells encapsulated into NF-hydrogels exhibited an upregulated release of anti-angiogenic factors (79\%), such as IGFBP-3, prolactin, angiostatin or vasohibin, rather than proangiogenic factors (21\%), such as FGF or IL8. This pattern was reversed for the RGD/REDV hydrogel, 
with $70 \%$ of the upregulated factors being proangiogenic (for example MCP-1, IGFBP-2, HB-EGF or VEGF). As VEGF is one of the most potent proangiogenic factors, Staubli et al. studied its release pattern and showed that VEGF was released into the supernatant of RGD/REDV hydrogels, whereas for NF hydrogels it was retained in the hydrogel and was not released into the supernatant. An in vivo assay in nude rats showed that RGD/REDV hydrogels supported blood vessel infiltration and were invaded by host vascularization. In addition to the lack of new vascularization, NF-hydrogels exhibited less host cell infiltration. Moreover, as the VGVAPG sequence attracts monocytes and is sensitive to proteolysis, RGD/REDV hydrogels showed degradation, thus allowing endothelial cells and fibroblasts to colonize the matrix [124].

Despite the difficulty of achieving vascular reconstruction, some groups are trying to go one step further and induce small-caliber vascular regeneration. Mahara et al., for example, have developed ELR-based scaffolds to be implanted into abdominal aortae in rats. An ELR-RGD was chosen in order to study the regeneration of blood vessel tissue. The hydrophobic sequence of the ELR, which is based on the VPGIG domain, starts to aggregate into particles up to $10 \mu \mathrm{m}$ in diameter above $12{ }^{\circ} \mathrm{C}$. In vitro assays showed that the RGD motif enhanced HUVEC adhesion to the scaffold, whereas this was not the case for the ELR alone. In the in vivo assay, blood vessel tissue regeneration was observed in rats that received the ELRRGD scaffold, whereas no regeneration was seen when an ELR scaffold was used. Moreover, regenerated tissues also exhibited connections with host tissue in the animals receiving ELR-RGD treatment. Histological analysis showed no calcium deposition or migration of mature $\mathrm{T}$ cells in the scaffolds, thus confirming that these ELR-based biomaterials are completely innocuous for the host [125].

Intracerebral hemorrhage (ICH), and the resulting brain edema, which causes damage to brain tissue, is one of the most common effects of many diseases. Indeed, patients who suffer ICH exhibit high morbidity and mortality rates [68]. As such, it is very important to develop both novel therapeutic strategies and new materials in order to treat the deleterious effects of ICH. In this regard, an RGD-containing ELR has been proposed to avoid the acute phase of intracerebral hemorrhage. To evaluate the treatment efficiency in in vivo assays, the hematoma volume and inflammatory response were analyzed. When comparing treatment with RGD alone or with RGD-ELR at six hours after the intracerebral hemorrhage, the hematoma volume was found to be lower for the group treated with the latter. This effect remained at 24 and 48 hours after the ICH. Thus, the RGD-containing ELR treatment reduced hematoma volume in a statistically significant manner. Moreover, celladhesion assays with b-End3 demonstrated that the RGDcontaining ELR treatment enhanced endothelial cell adhesion, as expected, with the injured region being covered and filled by the RGD-containing ELR. An infarction assay showed that the RGD-

containing ELR was stable and did not damage microvessels. Moreover, it blocked the leakage of blood components, especially IgG, from damaged vessels after ICH by coating them. Immunohistochemistry assays showed the presence of von Willebrand Factor (vWF), a key component of the coagulation system that is necessary for platelet adhesion to collagen. In addition, the RGD-containing ELR treatment resulted in significantly reduced overexpression of vWF and decreased the immune response as a consequence of postICH activated microglia [126].

\subsection{Tissue engineering future applications}

Although elastin-like biomaterials are already being used for in vivo tissue engineering purposes, new ELRs continue to be modified and optimized for increasing their performances. Numerous recent in vitro studies are being carried out to identify the best candidates. For example, the authors of a recent study prepared four different constructs mainly comprising the ELR sequence (APGVGV) 12 together with a cell-adhesion sequence (RGD) and a binding peptide called CBP for the binding and maintenance of mouse induced pluripotent stem cell (iPSCs). Upon coating untreated culture plates, the ELR construct containing both RGD and the CBP sequence (ERE-CBP) was the best in terms of mouse iPSC binding and expansion, and it was also demonstrated that the ability of the RGD sequence to allow cellular attachment was augmented by the CBP peptide, which had no effect when acting alone. Thus, cultures on ERE-CBP exhibited the typically shaped colonies of pluripotent cells. Moreover, expression of the pluripotent marker Oct $3 / 4$ and alkaline phosphatase activity on ERE-CBP was significantly higher than on the ERE alone. There were no significant differences between ERE-CBP and the construct lacking RGD. Thus, RGD showed its ability to enhance cell binding but not to maintain pluripotency, whereas CBP alone did not bind cells but maintained pluripotency at good levels. As such, the ERE-CBP results indicated the importance of the simultaneous presence of RGD and CBP for the maintenance of pluripotency. This new recombinant ECM protein could be the perfect candidate for use in tissue engineering in future in vivo experiments [127].

The formation of an extracellular matrix similar to the natural version is probably one of the main aims in tissue engineering. For this reason, one research group has studied fibers of an ELR containing a double-hydrophobic triblock structure also containing phenylalanine, known as GPG, in order to achieve cell adhesion and proliferation of embryonic mouse fibroblasts (NIH-3T3). The authors developed three different hydrophilic constructs, namely GPG, GPG-KAAK (where KAAK is a peptide that is known to increase celladhesion properties) and GPG-KAAK-GRGDS, which also contains the RGD motif. All these constructs were found to be excellent materials for coating polystyrene surfaces, with more than $90 \%$ remaining intact after washing with D-PBS. Although all three ELR constructs were able to form fibers at $37^{\circ} \mathrm{C}$, their behavior as cellular scaffolds differed markedly. Thus, when NIH-3T3 cells were cultured on the ELR, GPGKAAK-GRGDS allowed these cells to attach, forming wellspread, polygonal shapes. Indeed, after culture for three days, GPG-KAAK-GRGDS significantly promoted cell 
proliferation. In contrast, GPG only exhibited proliferation in a specific region, whereas GPG-KAAK did not support strong cell adhesion, probably as a result of hydrophobic interactions. Indeed, these two scaffolds showed worse cell adhesion than polystyrene plates. Thus, unexpectedly, KAAK did not enhance fibroblast adhesion. [128].

The creation of cytocompatible adhesives is another interesting approach within the tissue-engineering field. Thus, Brennan et al. developed an ELR (based on tyrosine, lysine and valine) called ELY16 and modified the tyrosine residues to 3,4-dihydroxyphenylalanine (DOPA) using the mushroom tyrosinase enzyme to form mELY16. Both ELY16 and mELY16 showed $>95 \%$ viability in NIH/3T3 fibroblasts, which is essential for a biomedical adhesive. Surprisingly, mELY16 exhibited stronger adsorption ability than glass coverslips. Both constructs (ELY and mELY16) exhibited greater adhesion strength than BSA and commercial sealant (>2MPa under dry conditions, with mELY16 giving a value of $0.24 \mathrm{MPa}$ under wet conditions). This new biomaterial could therefore be very useful in tissue engineering and regenerative medicine [129].

\section{CONCLUSION}

Different ELR-based constructs and their applications as advanced drug-delivery systems have been reviewed herein, and their ability to deploy therapeutic agents at specific sites in a safe and effective manner has been discussed.

Monomeric elastin like recombinamers have been analysed as soluble delivery systems when fused to therapeutic proteins in a recombinant manner, or as pharmacokinetic enhancers, either by chemical conjugation or by fusion protein expression of the drug. When used as therapeutic systems, these polypeptides are able to extend the half-life of pharmaceutical agents. Moreover, the incorporation of targeting peptides, such as receptor ligands or cell-penetrating domains, by genetic engineering allows an improved cellular uptake of constructs with a therapeutic function in damaged tissues. In addition, an appropriate selection of the amino acid sequence allows reactive sites that are useful for covalent chemical conjugation of molecules with demonstrated therapeutic activity to be included in the ELR chain, with chemicals and proteins being the most popular candidates. ELRs can also be designed to self-assemble in response to external stimuli, thus making them suitable for systemic delivery of a therapeutic drug and local delivery of the therapeutic agent into the damaged tissue [98]. Accumulation in tumor tissues after thermoresponsive transition of ELRs that are soluble at body temperature has been observed as a result of local overheating. Indeed, therapeutic hyperthermia has allowed the efficient local delivery of ELR conjugates into tumor tissues to be enhanced by taking advantage of tumor vasculature permeability and perfusion. Treatments including the synergy of more than one of these strategies have been used to improve the outcomes, especially in cancer therapy.

ELR-based nanoparticle formation, and the subsequent use thereof as drug nanocarriers, has also been reviewed.
Different strategies to induce polypeptide assembly have been described, including metal-ion induced self-assembly by means of a metal-chelating hexahistidine tag, which acts as a supramolecular crosslinker to induce NP formation when a divalent metal is added. The self-assembly properties of diblock monomeric ELRs allow the formation of structures such as nanoparticles, the reinforcement of which by interchain crosslinking to achieve more stable NPs has also been reviewed.

The biomedical applications of NPs have been discussed according to the different structures of the devices, including ELR-based nanoparticles that present growth factors which can interact with cells on their surfaces, some of which have shown promising results for the treatment of chronic skin wounds [98], induction of bone regeneration [121] or treatment of neuronal injuries [100]. Other NPs fused to proteins that are exposed on the surface of the NP and which have been used as therapeutic agents, for example lacritin nanoparticles to promote corneal wound healing and epithelial integrity, have also been discussed [123]. Furthermore, other NPs that can internalize a drug, therapeutic agent or even DNA such that the permeability and retention effect is enhanced, thereby increasing the exposure time of the drug and allowing an efficient delivery of poorly soluble drugs or the formation of polyplexes for gene therapy, have been reviewed. A combination of both strategies has also been applied to improve controlled drug delivery devices for application in advanced nanomedicine, especially for targeted cancer therapy, and may be applicable to a variety of anticancer drugs.

Finally, 3D ELR-based macrostructures, including depots, macromolecular carriers and hydrogels, which allow a highly localised treatment that is especially interesting for anticancer therapy, have been reviewed. Due to their ability to respond to changes in temperature, ELRs can aggregate to form injectable depots, which can be administered intratumorally, thereby facilitating localized anticancer drug exposure, maximizing drug retention within the tumor and improving treatments. Fused ELR-based depots can act as macromolecular carriers to deliver therapeutic agents such as glucagon-like peptide- 1 or curcumin, thereby representing a significant breakthrough in the treatment of type 2 diabetes and neuroinflammation therapies, respectively. The improvement achieved with the use of chemically and physically crosslinked hydrogels resides in their ability to be placed near the site of interest and deliver embedded drugs over a prolonged period of time locally, thereby minimizing any systemic effects. Particular attention has been paid to hydrogels containing a domain susceptible to proteolytic degradation, which allows sustained release of the therapeutic agent, as such systems could be a good model for controlled release of bioactive factors such as growth factors, hormones, antibodies, and other therapeutic macromolecules with potential future biomedical applications.

The use of ELR-based 3D macrostructures, especially hydrogels, in tissue engineering, with a particular focus on regeneration of the skeletal system, has also been reviewed. Thus, different approaches to obtain custom scaffolds made from amphiphilic ELRs that form hydrogels under 
physiological conditions and which mimic the part of the body to be treated, such as the skeletal system or vascular systems, or which can be used to regenerate soft tissues, have been discussed. Those biomimetic and innovative scaffolds are of particular importance for tissue engineering because of the possibility to integrate multiple bioactive motifs into the ELR sequences, or the ability to include appropriate cells for regenerating functional tissues. Studies of bicomponent supports, such as ELR-collagen, ELRsilicon substituted hydroxyapatite or ELR-hyaluronic acid hydrogel scaffolds, have shown the synergy that arises upon combination with ELRs, thus meaning that they should be promising candidates for tissue engineering.

\section{ACKNOWLEDGEMENTS}

The authors are grateful for financial support from the European Social Fund (ESF) and the European Regional Development Fund (ERDF), as well as funding from the EU (NMP-2014-646075, HEALTH-F4-2011-278557, PITN-GA2012-317306 and MSCA-ITN-2014-642687), the MINECO (MAT2015-68901-R, MAT2016-79435-R and MAT201678903-R), the JCyL (projects VA244U13 and VA313U14), the CIBER-BBN, the JCyL, the Instituto de Salud Carlos III under the "Network Center of Regenerative Medicine and Cellular Therapy of Castilla and Leon”.

\section{REFERENCES}

[1] Goldberg, M.; Langer, R.; Jia, X. Nanostructured materials for applications in drug delivery and tissue engineering. Journal of Biomaterials Science, Polymer Edition, 2007, 18(3), 241-268.

[2] Furth, M. E.; Atala, A.; Van Dyke, M. E. Smart biomaterials design for tissue engineering and regenerative medicine. Biomaterials, 2007, 28(34), 5068-5073.

[3] Vert, M., Biopolymers and artificial biopolymers in biomedical applications, an overview. In Biorelated Polymers, Springer: 2001; pp 63-79.

[4] Langer, R. New methods of drug delivery. Science, 1990, 1527-1533.

[5] Allen, T. M.; Cullis, P. R. Drug delivery systems: entering the mainstream. Science, 2004, 303(5665), 1818-1822.

[6] Lattin, J.; Belnap, D.; Pitt, W. Formation of eLiposomes as a drug delivery vehicle. Colloids and Surfaces B: Biointerfaces, 2012, 89, 93-100.

[7] Sarikaya, M.; Tamerler, C.; Jen, A. K.-Y.; Schulten, K.; Baneyx, F. Molecular biomimetics: nanotechnology through biology. Nature materials, 2003, 2(9), 577-585.

[8] Langer, R.; Tirrell, D. A. Designing materials for biology and medicine. Nature, 2004, 428(6982), 487-492.

[9] Zorlutuna, P.; Vrana, N. E.; Khademhosseini, A. The expanding world of tissue engineering: the building blocks and new applications of tissue engineered constructs. IEEE reviews in biomedical engineering, 2013, 6, 47-62.

[10] Girotti, A.; Orbanic, D.; Ibáñez-Fonseca, A.; Gonzalez-Obeso, C.; Rodríguez- Cabello, J. C. Recombinant Technology in the Development of Materials and Systems for Soft-Tissue Repair. Advanced healthcare materials, 2015, 4(16), 24232455.

[11] Floss, D. M.; Schallau, K.; Rose-John, S.; Conrad, U.; Scheller, J. Elastin-like polypeptides revolutionize recombinant protein expression and their biomedical application. Trends in biotechnology, 2010, 28(1), 37-45.

[12] Girotti, A.; Fernández-Colino, A.; López, I. M.; Rodríguez-Cabello, J. C.; Arias, F. J. Elastin- like recombinamers: Biosynthetic strategies and biotechnological applications. Biotechnology journal, 2011, 6(10), 1174-1186.

[13] Arias, F. J.; Santos, M.; Ibáñez-Fonseca, A.; Piña, M.; Serrano, S. Elastin-like recombinamers as smart drug delivery systems. Current drug targets, 2018, 19(4), 360-379.

[14] Meyer, D. E.; Chilkoti, A. Quantification of the effects of chain length and concentration on the thermal behavior of elastin-like polypeptides. Biomacromolecules, 2004, 5(3), 846-851.

[15] de Torre, I. G.; Wolf, F.; Santos, M.; Rongen, L.; Alonso, M.; Jockenhoevel, S.; Rodríguez-Cabello, J. C.; Mela, P. Elastin-like recombinamer-covered stents: Towards a fully biocompatible and nonthrombogenic device for cardiovascular diseases. Acta biomaterialia, 2015, 12, 146-155.

[16] Rodríguez-Cabello, J. C.; Arias, F. J.; Rodrigo, M. A.; Girotti, A. Elastin-like polypeptides in drug delivery. Advanced drug delivery reviews, 2016, 97, 85-100.

[17] MacEwan, S. R.; Chilkoti, A. Applications of elastin-like polypeptides in drug delivery. J Control Release, 2014, 190, 314-30.

[18] Arias, F. J.; Santos, M.; Fernandez-Colino, A.; Pinedo, G.; Girotti, A. Recent contributions of elastin-like recombinamers to biomedicine and nanotechnology. Curr Top Med Chem, 2014, 14(6), 819-36.

[19] Lyons, D. F.; Le, V.; Kramer, W. H.; Bidwell, G. L., 3rd; Lewis, E. A.; Raucher, D.; Correia, J. J. Effect of basic cell-penetrating peptides on the structural, thermodynamic, and hydrodynamic properties of a novel drug delivery vector, ELP[V5G3A2-150]. Biochemistry, 2014, 53(6), 1081-91.

[20] Kowalczyk, T.; Hnatuszko-Konka, K.; Gerszberg, A.; Kononowicz, A. K. Elastin-like polypeptides as a promising family of genetically-engineered protein based polymers. World J Microbiol Biotechnol, 2014, 30(8), 2141-52. 
[21] MacEwan, S. R.; Chilkoti, A. Controlled apoptosis by a thermally toggled nanoscale amplifier of cellular uptake. Nano Lett, 2014, 14(4), 2058-64.

[22] Jiang, T.; Zhang, Z.; Zhang, Y.; Lv, H.; Zhou, J.; Li, C.; Hou, L.; Zhang, Q. Dual-functional liposomes based on pH-responsive cell-penetrating peptide and hyaluronic acid for tumor-targeted anticancer drug delivery. Biomaterials, 2012, 33(36), 9246-58.

[23] Koren, E.; Torchilin, V. P. Cell-penetrating peptides: breaking through to the other side. Trends Mol Med, 2012, 18(7), 385-93.

[24] Milletti, F. Cell-penetrating peptides: classes, origin, and current landscape. Drug Discov Today, 2012, 17(15-16), 850-60.

[25] Dreher, M. R.; Raucher, D.; Balu, N.; Michael Colvin, O.; Ludeman, S. M.; Chilkoti, A. Evaluation of an elastin-like polypeptidedoxorubicin conjugate for cancer therapy. $J$ Control Release, 2003, 91(1-2), 31-43.

[26] Saxena, R.; Nanjan, M. J. Elastin-like polypeptides and their applications in anticancer drug delivery systems: a review. Drug Deliv, 2015, 22(2), 156-67.

[27] Walker, L.; Perkins, E.; Kratz, F.; Raucher, D. Cell penetrating peptides fused to a thermally targeted biopolymer drug carrier improve the delivery and antitumor efficacy of an acid-sensitive doxorubicin derivative. Int J Pharm, 2012, 436(1-2), 825-32.

[28] Moktan, S.; Perkins, E.; Kratz, F.; Raucher, D. Thermal targeting of an acid-sensitive doxorubicin conjugate of elastin-like polypeptide enhances the therapeutic efficacy compared with the parent compound in vivo. Mol Cancer Ther, 2012, 11(7), 1547-56.

[29] Moktan, S.; Ryppa, C.; Kratz, F.; Raucher, D. A thermally responsive biopolymer conjugated to an acid-sensitive derivative of paclitaxel stabilizes microtubules, arrests cell cycle, and induces apoptosis. Invest New Drugs, 2012, 30(1), 236-48.

[30] Sarangthem, V.; Cho, E. A.; Bae, S. M.; Singh, T. D.; Kim, S. J.; Kim, S.; Jeon, W. B.; Lee, B. H.; Park, R. W. Construction and application of elastin like polypeptide containing IL-4 receptor targeting peptide. PLoS One, 2013, 8(12).

[31] Hu, J.; Wang, G.; Liu, X.; Gao, W. Enhancing Pharmacokinetics, Tumor Accumulation, and Antitumor Efficacy by Elastin-Like Polypeptide Fusion of Interferon Alpha. Adv Mater, 2015, 27(45), 7320-4.

[32] Moktan, S.; Raucher, D. Anticancer activity of proapoptotic peptides is highly improved by thermal targeting using elastin-like polypeptides. Int J Pept Res Ther, 2012, 18(3), 227-237.

[33] Raucher, D.; Ryu, J. S. Cell-penetrating peptides: strategies for anticancer treatment. Trends $\mathrm{Mol}$ Med, 2015, 21(9), 560-70.

[34] Ryu, J. S.; Raucher, D. Anti-tumor efficacy of a therapeutic peptide based on thermo-responsive elastin-like polypeptide in combination with gemcitabine. Cancer Lett, 2014, 348(1-2), 177-84.

[35] Walker, L. R.; Ryu, J. S.; Perkins, E.; McNally, L. R.; Raucher, D. Fusion of cell-penetrating peptides to thermally responsive biopolymer improves tumor accumulation of p21 peptide in a mouse model of pancreatic cancer. Drug Des Devel Ther, 2014, 8, 1649-58.

[36] Mikecin, A. M.; Walker, L. R.; Kuna, M.; Raucher, D. Thermally targeted p21 peptide enhances bortezomib cytotoxicity in androgen-independent prostate cancer cell lines. Anticancer Drugs, 2014, 25(2), 189-99.

[37] Bidwell, G. L., 3rd; Perkins, E.; Raucher, D. A thermally targeted c-Myc inhibitory polypeptide inhibits breast tumor growth. Cancer Lett, 2012, 319(2), 136-43.

[38] Chade, A. R.; Tullos, N. A.; Harvey, T. W.; Mahdi, F.; Bidwell, G. L., 3rd Renal Therapeutic Angiogenesis Using a Bioengineered PolymerStabilized Vascular Endothelial Growth Factor Construct. J Am Soc Nephrol, 2016, 27(6), 1741-52.

[39] Verhoef, J. J.; Anchordoquy, T. J. Questioning the Use of PEGylation for Drug Delivery. Drug Deliv Transl Res, 2013, 3(6), 499-503.

[40] Nouri, F. S.; Wang, X.; Chen, X.; Hatefi, A. Reducing the Visibility of the Vector/DNA Nanocomplexes to the Immune System by ElastinLike Peptides. Pharm Res, 2015, 32(9), 3018-28.

[41] Meikle, S. T.; Pineiro, Y.; Banobre Lopez, M.; Rivas, J.; Santin, M. Surface functionalization superparamagnetic nanoparticles conjugated with thermoresponsive poly(epsilon-lysine) dendrons tethered with carboxybetaine for the mild hyperthermia-controlled delivery of VEGF. Acta Biomater, 2016, 40, 235-42.

[42] Yatvin, M.; Kreutz, W.; Horwitz, B.; Shinitzky, M. pH-sensitive liposomes: possible clinical implications. Science, 1980, 210(4475), 1253-1255. Callahan, D. J.; Liu, W.; Li, X.; Dreher, M. R.; Hassouneh, W.; Kim, M.; Marszalek, P.; Chilkoti, A. Triple stimulus-responsive polypeptide nanoparticles that enhance intratumoral spatial distribution. Nano letters, 2012, 12(4), 2165-2170.

[44] Han, W.; MacEwan, S. R.; Chilkoti, A.; López, G. P. Bio-inspired synthesis of hybrid silica nanoparticles templated from elastin-like polypeptide micelles. Nanoscale, 2015, 7(28), 12038-12044.

[45] Smits, F. C.; Castelijns, W. W.; van Hest, J. C. Crosslinked ELP-based nanoparticles, using the strain promoted azide-alkyne cycloaddition. European Polymer Journal, 2015, 62, 386-393.

[46] Smits, F.; Buddingh, B. C.; van Eldijk, M. B.; van Hest, J. Elastin-Like Polypeptide Based Nanoparticles: Design Rationale Toward Nanomedicine. Macromolecular bioscience, 2015, 15(1), 36-51. 
[47] Wang, W.; Despanie, J.; Shi, P.; Edman, M. C.; Lin, Y.-A.; Cui, H.; Heur, M.; Fini, M. E.; HammAlvarez, S. F.; MacKay, J. A. Lacritin-mediated regeneration of the corneal epithelia by protein polymer nanoparticles. Journal of Materials Chemistry B, 2014, 2(46), 8131-8141.

[48] Shi, P.; Aluri, S.; Lin, Y.-A.; Shah, M.; Edman, M.; Dhandhukia, J.; Cui, H.; MacKay, J. A. Elastinbased protein polymer nanoparticles carrying drug at both corona and core suppress tumor growth in vivo. Journal of controlled release, 2013, 171(3), 330-338.

[49] Nawroth, J. F.; McDaniel, J. R.; Chilkoti, A.; Jordan, R.; Luxenhofer, R. MaleimideFunctionalized Poly (2-Oxazoline) s and Their Conjugation to Elastin-Like Polypeptides. Macromolecular bioscience, 2016, 16(3), 322-333.

[50] Yeboah, A.; Cohen, R. I.; Faulknor, R.; Schloss, R.; Yarmush, M. L.; Berthiaume, F. The development and characterization of SDF1 $\alpha$-elastin-like-peptide nanoparticles for wound healing. Journal of Controlled Release, 2016, 232, 238-247.

[51] McCarthy, B.; Yuan, Y.; Koria, P. Elastin-likepolypeptide based fusion proteins for osteogenic factor delivery in bone healing. Biotechnol Prog, 2016, 32(4), 1029-37.

[52] Johnson, T.; Koria, P. Expression and purification of neurotrophin-elastin-like peptide fusion proteins for neural regeneration. BioDrugs, 2016, 30(2), 117-127.

[53] Iglesias, R.; Koria, P. Leveraging growth factor induced macropinocytosis for targeted treatment of lung cancer. Med Oncol, 2015, 32(12), 259.

[54] Sarangthem, V.; Kim, Y.; Singh, T. D.; Seo, B.-Y.; Cheon, S.-H.; Lee, Y.-J.; Lee, B.-H.; Park, R.-W. Multivalent Targeting Based Delivery of Therapeutic Peptide using AP1-ELP Carrier for Effective Cancer Therapy. Theranostics, 2016, 6(12), 2235.

[55] Janib, S. M.; Gustafson, J. A.; Minea, R. O.; Swenson, S. D.; Liu, S.; Pastuszka, M. K.; Lock, L. L.; Cui, H.; Markland, F. S.; Conti, P. S.; Li, Z.; MacKay, J. A. Multimeric disintegrin protein polymer fusions that target tumor vasculature. Biomacromolecules, 2014, 15(7), 2347-58.

[56] Zhao, P.; Dong, S.; Bhattacharyya, J.; Chen, M. iTEP nanoparticle-delivered salinomycin displays an enhanced toxicity to cancer stem cells in orthotopic breast tumors. Mol Pharm, 2014, 11(8), 2703-12.

[57] van Eldijk, M. B.; Schoonen, L.; Cornelissen, J. J.; Nolte, R. J.; van Hest, J. C. Metal Ion-Induced SelfAssembly of a Multi-Responsive Block Copolypeptide into Well-Defined Nanocapsules. Small, 2016, 12(18), 2476-83.

[58] Assal, Y.; Mizuguchi, Y.; Mie, M.; Kobatake, E. Growth Factor Tethering to Protein Nanoparticles via Coiled-Coil Formation for Targeted Drug Delivery. Bioconjug Chem, 2015, 26(8), 1672-7.

[59] Hu, J.; Xie, L.; Zhao, W.; Sun, M.; Liu, X.; Gao, W. Design of tumor-homing and $\mathrm{pH}$-responsive polypeptide-doxorubicin nanoparticles with enhanced anticancer efficacy and reduced side effects. Chem Commun (Camb), 2015, 51(57), 11405-8.

[60] Piña, M. J.; Girotti, A.; Santos, M.; RodriguezCabello, J. C.; Arias, F. J. Biocompatible ELRBased Polyplexes Coated with MUC1 Specific Aptamers and Targeted for Breast Cancer Gene Therapy. Mol Pharm, 2016, 13(3), 795-808.

[61] McDaniel, J. R.; Callahan, D. J.; Chilkoti, A. Drug delivery to solid tumors by elastin-like polypeptides. Advanced drug delivery reviews, 2010, 62(15), 1456-1467.

[62] Liu, W.; MacKay, J. A.; Dreher, M. R.; Chen, M.; McDaniel, J. R.; Simnick, A. J.; Callahan, D. J.; Zalutsky, M. R.; Chilkoti, A. Injectable intratumoral depot of thermally responsive polypeptideradionuclide conjugates delays tumor progression in a mouse model. Journal of controlled Release, 2010, 144(1), 2-9.

[63] Liu, W.; McDaniel, J.; Li, X.; Asai, D.; Quiroz, F. G.; Schaal, J.; Park, J. S.; Zalutsky, M.; Chilkoti, A. Brachytherapy using injectable seeds that are selfassembled from genetically encoded polypeptides in situ. Cancer research, 2012, 72(22), 5956-5965.

[64] Perera, M.; Krishnananthan, N.; Lindner, U.; Lawrentschuk, N. An update on focal therapy for prostate cancer. Nat Rev Urol, 2016, 13(11), 641653.

[65] Potters, L.; Torre, T.; Fearn, P. A.; Leibel, S. A.; Kattan, M. W. Potency after permanent prostate brachytherapy for localized prostate cancer. International Journal of Radiation Oncology* Biology* Physics, 2001, 50(5), 1235-1242.

[66] Stock, R. G.; Kao, J.; Stone, N. N. Penile erectile function after permanent radioactive seed implantation for treatment of prostate cancer. The Journal of urology, 2001, 165(2), 436-439.

[67] Qureshi, A. I.; Mendelow, A. D.; Hanley, D. F. Intracerebral haemorrhage. Lancet, 2009 , 373(9675), 1632-1644.

[68] Schaal, J. L.; Li, X.; Mastria, E.; Bhattacharyya, J.; Zalutsky, M. R.; Chilkoti, A.; Liu, W. Injectable polypeptide micelles that form radiation crosslinked hydrogels in situ for intratumoral radiotherapy. Journal of Controlled Release, 2016, 228, 58-66.

[69] Olthoff, C. M.; Schouten, J. S.; van de Borne, B. W.; Webers, C. A. Noncompliance with ocular hypotensive treatment in patients with glaucoma or ocular hypertension: an evidence-based review. Ophthalmology, 2005, 112(6), 953-961. e7.

[70] Shukla, P.; Kumar, M.; Keshava, G. Mycotic keratitis: an overview of diagnosis and therapy. Mycoses, 2008, 51(3), 183-199. 
[71] Karnati, R.; Laurie, D. E.; Laurie, G. W. Lacritin and the tear proteome as natural replacement therapy for dry eye. Experimental eye research, 2013, 117, 39-52.

[72] Wang, W.; Jashnani, A.; Aluri, S. R.; Gustafson, J. A.; Hsueh, P.-Y.; Yarber, F.; McKown, R. L.; Laurie, G. W.; Hamm-Alvarez, S. F.; MacKay, J. A. A thermo-responsive protein treatment for dry eyes. Journal of Controlled Release, 2015, 199, 156-167.

[73] Shamji, M. F.; Chen, J.; Friedman, A. H.; Richardson, W. J.; Chilkoti, A.; Setton, L. A. Synthesis and characterization of a thermallyresponsive tumor necrosis factor antagonist. Journal of Controlled Release, 2008, 129(3), 179186.

[74] Kimmerling, K.; Furman, B.; Mangiapani, D.; Moverman, M.; Sinclair, S.; Huebner, J.; Chilkoti, A.; Kraus, V. B.; Setton, L.; Guilak, F. Sustained intra-articular delivery of IL-1RA from a thermallyresponsive elastin-like polypeptide as a therapy for post-traumatic arthritis. European cells \& materials, 2015, 29, 124.

[75] Manandhar, B.; Ahn, J.-M. Glucagon-like peptide-1 (GLP-1) analogs: recent advances, new possibilities, and therapeutic implications. J. Med. Chem, 2015, 58(3), 1020-1037.

[76] Amiram, M.; Luginbuhl, K.; Li, X.; Feinglos, M.; Chilkoti, A. A depot-forming glucagon-like peptide-1 fusion protein reduces blood glucose for five days with a single injection. Journal of Controlled Release, 2013, 172(1), 144-151.

[77] Shamji, M. F.; Whitlatch, L.; Friedman, A. H.; Richardson, W. J.; Chilkoti, A.; Setton, L. A. An injectable and in situ-gelling biopolymer for sustained drug release following perineural administration. Spine, 2008, 33(7), 748.

[78] Sinclair, S. M.; Bhattacharyya, J.; McDaniel, J. R.; Gooden, D. M.; Gopalaswamy, R.; Chilkoti, A.; Setton, L. A. A genetically engineered thermally responsive sustained release curcumin depot to treat neuroinflammation. Journal of Controlled Release, 2013, 171(1), 38-47.

[79] Wang, X.; Kluge, J. A.; Leisk, G. G.; Kaplan, D. L. Sonication-induced gelation of silk fibroin for cell encapsulation. Biomaterials, 2008, 29(8), 10541064.

[80] Drury, J. L.; Mooney, D. J. Hydrogels for tissue engineering: scaffold design variables and applications. Biomaterials, 2003, 24(24), 43374351.

[81] Amruthwar, S. S.; Janorkar, A. V. Preparation and characterization of elastin-like polypeptide scaffolds for local delivery of antibiotics and proteins. Journal of Materials Science: Materials in Medicine, 2012, 23(12), 2903-2912.

[82] Mecham, R. P.; Broekelmann, T. J.; Fliszar, C. J.; Shapiro, S. D.; Welgus, H. G.; Senior, R. M. Elastin degradation by matrix metalloproteinases Cleavage site specificity and mechanisms of elastolysis. Journal of Biological Chemistry, 1997, 272(29), 18071-18076.

[83] Bandiera, A.; Markulin, A.; Corich, L.; Vita, F.; Borelli, V. Stimuli-induced release of compounds from elastin biomimetic matrix. Biomacromolecules, 2013, 15(1), 416-422.

[84] Asai, D.; Xu, D.; Liu, W.; Quiroz, F. G.; Callahan, D. J.; Zalutsky, M. R.; Craig, S. L.; Chilkoti, A. Protein polymer hydrogels by in situ, rapid and reversible self-gelation. Biomaterials, 2012, 33(21), 5451-5458.

[85] Greish, K.; Frandsen, J.; Scharff, S.; Gustafson, J.; Cappello, J.; Li, D.; O'Malley, B. W.; Ghandehari, H. Silk-elastinlike protein polymers improve the efficacy of adenovirus thymidine kinase enzyme prodrug therapy of head and neck tumors. The journal of gene medicine, 2010, 12(7), 572-579.

[86] Gustafson, J.; Greish, K.; Frandsen, J.; Cappello, J.; Ghandehari, H. Silk-elastinlike recombinant polymers for gene therapy of head and neck cancer: from molecular definition to controlled gene expression. Journal of Controlled Release, 2009, 140(3), 256-261.

[87] Huang, W.; Rollett, A.; Kaplan, D. L. Silk-elastinlike protein biomaterials for the controlled delivery of therapeutics. Expert opinion on drug delivery, 2015, 12(5), 779-791.

[88] Fernandez-Colino A,; Quinteros D. A.; Allemandi D. A.; Girotti A.; Palma S. D.; Arias F. J. SelfAssembling Elastin-Like Hydrogels for Timolol Delivery: Development of an Ophthalmic Formulation Against Glaucoma. Molecular pharmaceutics, 2017, 14(12), 4498-4508.

[89] Gustafson, J. A.; Ghandehari, H. Silk-elastinlike protein polymers for matrix-mediated cancer gene therapy. Advanced drug delivery reviews, 2010, 62(15), 1509-1523.

[90] Price, R.; Poursaid, A.; Cappello, J.; Ghandehari, H. In vivo evaluation of matrix metalloproteinase responsive silk-elastinlike protein polymers for cancer gene therapy. Journal of Controlled Release, 2015, 213, 96-102.

[91] Chvapil, M.; Owen, J.; Clark, D.; Koorajian, S.; Goodman, A. Effect of collagen crosslinking on the rate of resorption of implanted collagen tubing in rabbits. Journal of biomedical materials research, 1977, 11(2), 297-314.

[92] Rosa, A. L.; De Oliveira, P. T.; Beloti, M. M. Macroporous scaffolds associated with cells to construct a hybrid biomaterial for bone tissue engineering. Expert review of medical devices, 2008, 5(6), 719-728.

[93] Kyle, S.; Aggeli, A.; Ingham, E.; McPherson, M. J. Production of self-assembling biomaterials for tissue engineering. Trends in biotechnology, 2009, 27(7), 423-433. 
[94] Yannas, I.; Burke, J. F. Design of an artificial skin. I. Basic design principles. Journal of biomedical materials research, 1980, 14(1), 65-81.

[95] Amruthwar, S. S.; Puckett, A. D.; Janorkar, A. V. Preparation and characterization of novel elastinlike polypeptide-collagen composites. Journal of Biomedical Materials Research Part A, 2013, 101(8), 2383-2391.

[96] Anderson, T. R.; Marquart, M. E.; Janorkar, A. V. Effective release of a broad spectrum antibiotic from elastin-like polypeptide- collagen composite. Journal of Biomedical Materials Research Part A, 2015, 103(2), 782-790.

[97] Mano, J. F.; Silva, G. A.; Azevedo, H. S.; Malafaya, P. B.; Sousa, R. A.; Silva, S. S.; Boesel, L. F.; Oliveira, J. M.; Santos, T. C.; Marques, A. P.; Neves, N. M.; Reis, R. L. Natural origin biodegradable systems in tissue engineering and regenerative medicine: present status and some moving trends. J R Soc Interface, 2007, 4(17), 9991030.

[98] Williams, D. J.; Sebastine, I. M. Tissue engineering and regenerative medicine: manufacturing challenges. IEE proceedings. Nanobiotechnology, 2005, 152(6), 207-10.

[99] Webber, M. J.; Appel, E. A.; Meijer, E.; Langer, R. Supramolecular biomaterials. Nature materials, 2016, 15(1), 13-26.

[100] Urry, D. W., What sustains life? Consilient mechanisms for protein-based machines and materials. Springer-Verlag: New York, 2006.

[101] Carlos Rodriguez-Cabello, J.; Martin, L.; Girotti, A.; Garcia-Arevalo, C.; Javier Arias, F.; Alonso, M. Emerging applications of multifunctional elastinlike recombinamers. Nanomedicine, 2011, 6(1), 111-122.

[102] Girotti, A.; Reguera, J.; Rodríguez-Cabello, J. C.; Arias, F. J.; Alonso, M.; Testera, A. M. Design and bioproduction of a recombinant multi(bio)functional elastin-like protein polymer containing cell adhesion sequences for tissue engineering purposes. Journal of Materials ScienceMaterials in Medicine, 2004, 15(4), 479-484.

[103] Corich, L.; Busetti, M.; Petix, V.; Passamonti, S.; Bandiera, A. Evaluation of a biomimetic 3D substrate based on the Human Elastin-like Polypeptides (HELPs) model system for elastolytic activity detection. J Biotechnol, 2017, 255, 57-65.

[104] Li, Y.; Rodriguez-Cabello, J. C.; Aparicio, C. Intrafibrillar Mineralization of Self-Assembled Elastin-Like Recombinamer Fibrils. ACS Applied Materials \& Interfaces, 2017, 9(7), 5838-5846.

[105] Raphel, J.; Karlsson, J.; Galli, S.; Wennerberg, A.; Lindsay, C.; Haugh, M. G.; Pajarinen, J.; Goodman, S. B.; Jimbo, R.; Andersson, M.; Heilshorn, S. C. Engineered protein coatings to improve the osseointegration of dental and orthopaedic implants. Biomaterials, 2016, 83, 269-82.
[106] Gonzalez, M.; Salvagni, E.; Rodriguez-Cabello, J. C.; Ruperez, E.; Gil, F. J.; Pena, J.; Manero, J. M. A low elastic modulus Ti-Nb-Hf alloy bioactivated with an elastin-like protein-based polymer enhances osteoblast cell adhesion and spreading. Journal of Biomedical Materials Research Part A, 2013, 101A(3), 819-826.

[107] Ozturk, N.; Girotti, A.; Kose, G. T.; RodríguezCabello, J. C.; Hasirci, V. Dynamic cell culturing and its application to micropatterned, elastin-like protein-modified poly(N-isopropylacrylamide) scaffolds. Biomaterials, 2009, 30(29), 5417-5426.

[108] Betre, H.; Setton, L.; Meyer, D.; Chilkoti, A. Characterization of a genetically engineered elastinlike polypeptide for cartilaginous tissue repair. Biomacromolecules, 2002, 3(5), 910-916.

[109] Liu, J. C.; Heilshorn, S. C.; Tirrell, D. A. Comparative cell response to artificial extracellular matrix proteins containing the RGD and CS5 cellbinding domains. Biomacromolecules, 2004, 5(2), 497-504.

[110] de Torre, I. G.; Wolf, F.; Santos, M.; Rongen, L.; Alonso, M.; Jockenhoevel, S.; Rodriguez-Cabello, J. C.; Mela, P. Elastin-like recombinamer-covered stents: Towards a fully biocompatible and nonthrombogenic device for cardiovascular diseases. Acta biomaterialia, 2015, 12, 146-55.

[111] Dimitriou, R.; Jones, E.; McGonagle, D.; Giannoudis, P. V. Bone regeneration: current concepts and future directions. BMC medicine, 2011, 9, 66.

[112] Gong, T.; Xie, J.; Liao, J.; Zhang, T.; Lin, S.; Lin, Y. Nanomaterials and bone regeneration. Bone research, 2015, 3, 15029.

[113] Coletta, D. J.; Ibáñez-Fonseca, A.; Missana, L. R.; Jammal, M. V.; Vitelli, E. J.; Aimone, M.; Zabalza, F.; Issa, J. P. M.; Alonso, M.; Rodríguez-Cabello, J. C. Bone regeneration mediated by a bioactive and biodegradable ECM-like hydrogel based on elastinlike recombinamers. Tissue Engineering, 2017, (ja).

[114] McCarthy, B.; Yuan, Y.; Koria, P. Elastin-likepolypeptide based fusion proteins for osteogenic factor delivery in bone healing. Biotechnology progress, 2016, 32(4), 1029-1037.

[115] Vila, M.; García, A.; Girotti, A.; Alonso, M.; Rodríguez-Cabello, J. C.; González-Vázquez, A.; Planell, J. A.; Engel, E.; Buján, J.; GarcíaHonduvilla, N. 3D silicon doped hydroxyapatite scaffolds decorated with Elastin-like Recombinamers for bone regenerative medicine. Acta biomaterialia, 2016, 45, 349-356.

[116] Shuturminska, K.; Tarakina, N. V.; Azevedo, H. S.; Bushby, A. J.; Mata, A.; Anderson, P.; Al-Jawad, M. Elastin-like protein, with statherin derived peptide, controls fluorapatite formation and morphology. Frontiers in Physiology, 2017, 8.

[117] Glassman, M. J.; Avery, R. K.; Khademhosseini, A.; Olsen, B. D. Toughening of thermoresponsive 
arrested networks of elastin-like polypeptides to engineer cytocompatible tissue scaffolds. Biomacromolecules, 2016, 17(2), 415-426.

[118] Gurumurthy, B.; Bierdeman, P. C.; Janorkar, A. V. Spheroid model for functional osteogenic evaluation of human adipose derived stem cells. Journal of Biomedical Materials Research Part A, 2017, 105(4), 1230-1236.

[119] Gurumurthy, B.; Bierdeman, P. C.; Janorkar, A. V. Composition of elastin like polypeptide-collagen composite scaffold influences in vitro osteogenic activity of human adipose derived stem cells. Dental Materials, 2016, 32(10), 1270-1280.

[120] Fahy, N.; Alini, M.; Stoddart, M. J. Mechanical stimulation of mesenchymal stem cells: Implications for cartilage tissue engineering. Journal of orthopaedic research : official publication of the Orthopaedic Research Society, 2017.

[121] Zhu, D.; Wang, H.; Trinh, P.; Heilshorn, S. C.; Yang, F. Elastin-like protein-hyaluronic acid (ELPHA) hydrogels with decoupled mechanical and biochemical cues for cartilage regeneration. Biomaterials, 2017, 127, 132-140.

[122] Bertassoni, L. E.; Cecconi, M.; Manoharan, V.; Nikkhah, M.; Hjortnaes, J.; Cristino, A. L.; Barabaschi, G.; Demarchi, D.; Dokmeci, M. R.; Yang, Y.; Khademhosseini, A. Hydrogel Bioprinted Microchannel Networks for Vascularization of Tissue Engineering Constructs. Lab on a chip, 2014, 14(13), 2202-2211.

[123] Weber, M.; Gonzalez de Torre, I.; Moreira, R.; Frese, J.; Oedekoven, C.; Alonso, M.; Rodriguez Cabello, C. J.; Jockenhoevel, S.; Mela, P. MultipleStep Injection Molding for Fibrin-Based TissueEngineered Heart Valves. Tissue Engineering. Part C, Methods, 2015, 21(8), 832-840.

[124] Staubli, S. M.; Cerino, G.; De Torre, I. G.; Alonso, M.; Oertli, D.; Eckstein, F.; Glatz, K.; Cabello, J. C. R.; Marsano, A. Control of angiogenesis and host response by modulating the cell adhesion properties of an Elastin-Like Recombinamer-based hydrogel. Biomaterials, 2017, 135, 30-41.

[125] Mahara, A.; Kiick, K. L.; Yamaoka, T. In vivo guided vascular regeneration with a non-porous elastin-like polypeptide hydrogel tubular scaffold. Journal of Biomedical Materials Research Part A, 2017, 105(6), 1746-1755.

[126] Park, J.; Kim, J. Y.; Choi, S.-K.; Kim, J. Y.; Kim, J. H.; Jeon, W. B.; Lee, J. E. Thermo-sensitive assembly of the biomaterial REP reduces hematoma volume following collagenase-induced intracerebral hemorrhage in rats. Nanomedicine: Nanotechnology, Biology and Medicine, 2017.

[127] Adnan, N.; Mie, M.; Haque, A.; Hossain, S.; Mashimo, Y.; Akaike, T.; Kobatake, E. Construction of a Defined Biomimetic Matrix for Long-Term Maintenance of Mouse Induced
Pluripotent Stem Cells. Bioconjugate chemistry, 2016, 27(7), 1599-1605.

[128] Le, D. H.; Tsutsui, Y.; Sugawara-Narutaki, A.; Yukawa, H.; Baba, Y.; Ohtsuki, C. Doublehydrophobic elastin-like polypeptides with added functional motifs: Self-assembly and cytocompatibility. Journal of Biomedical Materials Research Part A, 2017.

[129] Brennan, M. J.; Kilbride, B. F.; Wilker, J. J.; Liu, J. C. A bioinspired elastin-based protein for a cytocompatible underwater adhesive. Biomaterials, 2017, 124, 116-125. 